\title{
ITRAQ-Based Proteomics Analysis of Triptolide On Human A549 Lung Adenocarcinoma Cells
}

\author{
Fangqiong Lia Dongxiao Zhao ${ }^{\mathrm{a}} \quad$ Suwen Yang ${ }^{\mathrm{b}} \quad$ Juan Wang ${ }^{\mathrm{a}}$ Qin Liu ${ }^{\mathrm{a}} \quad$ Xin Jin ${ }^{\mathrm{a}}$ \\ Wei Wanga \\ aDepartment of Clinical Laboratory, Tongde Hospital of Zhejiang Province, Hangzhou, ${ }^{b}$ Department of \\ Clinical Laboratory, Sir Run Run Shaw Hospital, Medical College, Zhejiang University, Hangzhou, P.R. \\ China
}

\section{Key Words}

Triptolide $・$ NSCLC $・$ ITRAQ $・$ Proteome

\begin{abstract}
Background/Aims: Triptolide (TP) is a diterpenoid triepoxide extracted from the traditional Chinese medical herb Tripterygium wilfordii that exerts prominent broad-spectrum anticancer activity to repress proliferation and induce cancer cell apoptosis through various molecular pathways. We previously observed that TP inhibits the progression of A549 cells and pancreatic cancer cells (PNCA-1) in vitro. However, the complex molecular mechanism underlying the anticancer activity of TP is not well understood. Methods: To explore the molecular mechanisms by which TP induces lung cancer cell apoptosis, we investigated changes in the protein profile of A549 cells treated with TP using a proteomics approach (iTRAQ [isobaric tags for relative and absolute quantitation] combined with NanoLC-MS/MS [nano liquid chromatographymass spectrometry]). Changes in the profiles of the expressed proteins were analyzed using the bioinformatics tools OmicsBean and the Kyoto Encyclopedia of Genes and Genomes (KEGG) and were verified using western blotting. Apoptosis and cell cycle effects were analyzed using flow cytometry. Results: TP induced apoptosis in A549 cells and blocked A549 cells at the G2/M phase. Using iTRAQ technology, we observed 312 differentially expressed proteins associated in networks and implicated in different KEGG pathways. Gene Ontology (GO) analysis showed the overviews of dysregulated proteins in the biological process (BP), cell component (CC), and molecular function (MF) categories. Moreover, some candidate proteins involved in PARP1/AIF and nuclear Akt signaling pathways or metastasis processes were validated by western blotting. Conclusion: TP exerted anti-tumor activity on non-small cell lung cancer (NSCLC) A549 lung adenocarcinoma cells by dysregulating tumor-related protein expression. Herein, we provide a preliminary study of TP-related cytotoxicity on A549 cells using proteomics tools. These findings may improve the current understanding of the anti-tumor effects of TP on lung cancer cells and may reveal candidate proteins as potential targets for the treatment of lung cancer.




\section{Cellular Physiology Cell Physiol Biochem 2018;45:917-934 \begin{tabular}{ll|l} 
DOI: 10.1159/000487286 & (0) 2018 The Author(s). Published by S. Karger AG, Basel \\
and Biochemistry & Published online: February 08, 2018 www.karger.com/cpb
\end{tabular} \\ Li et al.: Proteomics Analysis of Triptolide Targets in A549 Cells}

\section{Introduction}

Lung cancer is one of the most prevalent malignancies in humans, and its incidence and mortality are increasing worldwide [1]. Approximately $80 \%$ of lung cancers are nonsmall cell lung cancers (NSCLCs). Although diagnostic and treatment methods have recently improved markedly, no significant improvements in the prognosis of patients with NSCLC have been achieved, as the 5-year survival rates for all patients diagnosed with lung cancer remain at approximately $15 \%[2,3]$. Currently, the major methods for lung cancer treatment include surgery, chemotherapy and radiotherapy. Among these methods, chemotherapy can significantly relieve symptoms and improve patient quality of life. However, the efficacy and safety of this treatment remain a primary concern. Chemotherapy drugs have some serious side effects, and the toxicity of these drugs has always been an obstacle to clinical applications [4]. Therefore, the development of new therapeutic drugs for lung cancer that have high efficacy and low toxicity is clinically important.

Triptolide (TP), originally extracted from the traditional Chinese medicinal plant Tripterygium wilfordii [5], has been confirmed to have myriad biological properties, including immunosuppression and anti-inflammatory effects, and has been applied for the treatment of autoimmune diseases, such as nephritis and rheumatoid arthritis [6-8]. Recently, numerous studies have demonstrated that TP possesses prominent anti-tumor activities in diverse tumor cell types in vitro, such as breast [9], pancreatic [10], ovarian [11], lung [12], and prostate cancers [13]. TP can also prevent tumor growth in vivo via cell proliferation inhibition and apoptosis induction [14]. Moreover, studies have reported that TP sensitizes human cancer cells to cisplatin, 5-fluorouracil (5-FU) and TNF-alpha-induced apoptosis in vivo and in vitro $[15,16]$. In a previous study, we observed that TP induces apoptosis in human lung cancer cells through PP2A-regulated ERK, p38, MAPK and Akt signaling pathways [17]. Until recently, the activity of TP in inducing tumor cell death has been well documented, but the complex molecular targets of TP anti-tumor activity have not been well characterized. Thus, a powerful tool to accurately monitor and quantitatively detect changes in protein expression in response to TP treatment is needed.

Proteomics approaches, enabling relatively comprehensive global analyses, have been widely used to examine complex biological functions [18-20]. The isobaric tags for the relative and absolute quantitation (iTRAQ) method combined with nano liquid chromatographymass spectrometry (NanoLC-MS/MS), developed for protein quantitation, represent a high-throughput quantitative technique with high sensitivity and reproducibility. Currently, iTRAQ-based proteomics has been widely used to investigate the mechanistic effects of chemicals on cancer [21-24].

In the present study, we employed a strategy combining iTRAQ with NanoLC-MS/MS to analyze alterations in the protein profile of the A549 lung adenosquamous carcinoma cell line following TP treatment. Differential protein expression data may provide a valuable resource to reveal potential molecular targets underlying the anticancer activity of TP and to improve the understanding of the anti-tumor effects of TP on lung cancer.

\section{Materials and Methods}

\section{Cell culture and treatment}

Human lung cancer A549 cells (American Type Culture Collection; ATCC CCL185) were maintained in monolayer culture at $37^{\circ} \mathrm{C}$ in a humidified atmosphere with $5 \% \mathrm{CO}_{2}$ in RPMI-1640 medium (Gibco-BRL, USA) supplemented with $10 \%$ fetal bovine serum (FBS) (Sijiqin Biotechnology Co. Ltd., China) and $1 \%$ penicillin/streptomycin solution (100 U/ml penicillin and $100 \mu \mathrm{g} / \mathrm{ml}$ streptomycin). A total of $20 \mathrm{mg}$ of TP (purity $\geq 98 \%$, Beijing Fan-China Biotechnology Co., Ltd.) was dissolved in $0.5 \mathrm{ml}$ dimethylsulfoxide (DMSO) to obtain a $100 \%$ stock solution, which was subsequently stored at $-20^{\circ} \mathrm{C}$ and diluted with medium prior to use in experiments. The final DMSO concentration did not exceed $0.1 \%(\mathrm{v} / \mathrm{v})$ throughout the study. For 


\section{Cellular Physiology Cell Physiol Biochem 2018;45:917-934 and Biochemistry Published \begin{tabular}{l|l} 
DOI: 10.1159/000487286 & $\begin{array}{l}\text { C } 2018 \text { The Author(s). Published by S. Karger AG, Basel } \\
\text { www.karger.com/cpb }\end{array}$
\end{tabular}}

Li et al.: Proteomics Analysis of Triptolide Targets in A549 Cells

exposure experiments, A549 cells at approximately $80 \%$ confluency were transferred to medium containing $12.5,50$, and $200 \mathrm{ng} / \mathrm{ml}$ of TP and were cultured for $36 \mathrm{~h}$ (24-h 50\% inhibitory concentration [IC50]=273.0 $\mathrm{ng} / \mathrm{ml}$ ) [17]. Cells treated with an equal amount of DMSO were employed as a control, and all of the treatments were performed in triplicate. Three replicate proteomics analyses were performed for each test concentration and the control group. The iTRAQ experimental results described herein are only for the 200 $\mathrm{ng} / \mathrm{ml} \mathrm{TP}$ concentration, as cells represented more acute TP cytotoxicity at this concentration.

\section{Flow cytometric cell cycle and cell apoptosis analysis}

The effects of TP on cell cycle progression were measured using flow cytometry. The fixed cells were stained with propidium iodide (PI, ComWin Biotech Co. Ltd., China) solution (50 $\mu \mathrm{g} / \mathrm{ml}$ PI and $100 \mu \mathrm{g} / \mathrm{ml}$ RNase A in PBS) and were subsequently subjected to cell cycle analysis. Cell apoptosis was measured using Annexin V/PI double staining (ComWin Biotech Co. Ltd., China). Briefly, $100 \mu$ l of binding buffer containing $2.5 \mu \mathrm{l}$ of Annexin V-fluorescein isothiocyanate (FITC) and $1 \mu \mathrm{l}$ of PI was added to the cell suspension, followed by incubation for $30 \mathrm{~min}$ in the dark. The samples were assayed using a Beckman-Coulter Flow Cytometer with excitation at $488 \mathrm{~nm}$ and emission at $525 \mathrm{~nm}$ for FITC and $575 \mathrm{~nm}$ for PI. The data were analyzed using FlowJo software.

\section{Protein preparation}

The harvested cells were washed five times using ice-cold PBS and disrupted using enhanced RIPA lysis buffer (Beyotime Co., China) containing protease and phosphatase inhibitors for $30 \mathrm{~min}$ on ice, followed by ten cycles of 5 -second bursts of sonication with 30 -second intervals. The cell debris was removed by centrifugation at $12,000 \times \mathrm{g}$ for $30 \mathrm{~min}$ at $4^{\circ} \mathrm{C}$, and the supernatants were collected. Protein concentrations were assayed using a bicinchoninic acid (BCA) protein assay kit according to the manufacturer's instructions (Beyotime Co., China). Bovine serum albumin (BSA) was used as the standard.

\section{iTRAQ labeling and high-pH RPLC fractionation}

The iTRAQ Reagent 4-Plex kit (AB Sciex, USA) was used according to the manufacturer's instructions to label peptides. Equal amounts of protein $(100 \mu \mathrm{g}$ per sample) obtained from TP-treated and control cells were labeled using iTRAQ labeling reagents. The TP-treated samples were labeled using 117, while the control samples were labeled using 114. Briefly, the proteins in each sample were reduced with dithiothreitol (DTT) and were subsequently alkylated with iodoacetamide. The samples were digested overnight at $37^{\circ} \mathrm{C}$ using trypsin (AB Sciex, USA) at a trypsin:protein ratio of 1:20 (W/W). The tryptic peptides were labeled using iTRAQ reagents. The labeled samples were combined and lyophilized. The peptide mixtures were dissolved in high-pH reverse phase (HP-RP) solvent A (20 mM ammonium formate, pH 10.0). The peptides were fractionated using the Shimadzu LC-30A system with a Durashell-C18 column $(4.6 \mathrm{~mm} \times 250 \mathrm{~mm}, 5 \mu \mathrm{m} 100$ $\AA$, Agela, China) for high-pH RP chromatography. A total of 40 RP fractions were collected and subsequently dried and reconstituted using $30 \mu \mathrm{l}$ of $0.1 \%$ FA for NanoLC-MS/MS analysis.

\section{NanoLC-MS/MS analysis}

Separation was performed using the Eksigent nanoLC-Ultra ${ }^{\mathrm{TM}}$ 2D System combined with the cHiPLC ${ }^{\mathrm{TM}}$ Nanoflex system in Trap-Elute mode connected to a Triple TOF 4600 mass spectrometer (AB Sciex, USA). Briefly, $8 \mu \mathrm{l}$ of each fraction was loaded onto the cHiPLC trap $(200 \mu \mathrm{m} \times 500 \mu \mathrm{m}$ ChromXP C18-CL $3 \mu \mathrm{m}$ $300 \AA)$ and washed for $15 \mathrm{~min}$ at $2 \mu \mathrm{l} / \mathrm{min}$. Subsequently, an elution gradient of $10-43 \%$ acetonitrile $(0.1 \%$ formic acid) in an 85-min gradient at $300 \mathrm{nl} / \mathrm{min}$ was used on a nano cHiPLC column (75 $\mu \mathrm{m} \times 15 \mathrm{~cm}$ ChromXP C18-CL $3 \mu \mathrm{m} 300 \AA$ ). The MS analysis was performed using a nano ion spray voltage maintained at $2.3 \mathrm{kV}$ and a scan range of 350 to $1500(\mathrm{~m} / \mathrm{z})$ in the positive-ion mode. Full-scan MS spectra were acquired from 40 precursors selected for MS/MS from an m/z 100-1500 range using a dynamic exclusion setting of $30 \mathrm{~s}$. The IDA CE parameter script, which selected up to 40 precursors with charge states of $2+$ to $4+$, automatically controlled the collision energy (CE). The mass spectrometer was calibrated using the tryptic peptides of beta-galactosidase.

Protein identification and quantitation

Peptide identification and quantification were conducted using ProteinPilot 4.2 software (AB Sciex, USA). The following search parameters were used: (1) sample type, iTRAQ 4-plex (Peptide Labeled); (2) 


\section{Cellular Physiology Cell Physiol Biochem 2018;45:917-934

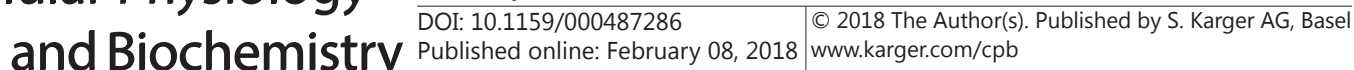

Li et al.: Proteomics Analysis of Triptolide Targets in A549 Cells

cysteine alkylation, iodoacetamide; (3) digestion, trypsin; (4) instrument, Triple TOF 4600; (5) special factors, none; (6) species, Homo sapiens; (7) ID Focus, biological modifications; (8) database, UniProtKB/ Swiss-Prot FASTA; and (9) search effort, Thorough ID. In the iTRAQ quantitation, the Pro Group algorithm was automatically selected to calculate the reporter-peak areas. To estimate the false discovery rate (FDR) for peptide identification, a decoy database search strategy was adopted. For this study, a strict unused confidence score of $>1.3$ was used as the qualification criterion, corresponding to a peptide confidence level of $95 \%$. If the iTRAQ ratios were $>2.0$ or $<0.5$ in the samples obtained from the TP-treated A549 cells relative to those of the control group, then the proteins were considered differentially expressed.

\section{Bioinformatics analysis of proteomics data}

The identified proteins were classified according to annotations from the UniProt knowledge base (Swissprot/TrEMBL, http://www.uniprot.org/).The multi-omics data analysis tool, OmicsBean, was used to analyze the obtained proteomics data (http://www.omicsbean.com), in which distributions in biological process (BP), cellular components (CCs) and molecular functions (MF) were assigned to each protein based on Gene Ontology (GO) categories. Kyoto Encyclopedia of Genes and Genomes (KEGG) pathway analysis (http://www.genome.ad.jpkegg/pathway.html) was performed to enrich high-level functions in the defined biological systems. Protein-protein interaction (PPI) analysis was performed using Cytoscape software, with a confidence cutoff of 400; interactions with larger confident scores are indicated with solid lines between genes/proteins, or otherwise shown as dashed lines.

\section{Western blotting}

The A549 cells were washed twice with cold PBS and lysed in $200 \mu$ RIPA lysis buffer (50 mM Tris$\mathrm{HCl}, 150 \mathrm{mM} \mathrm{NaCl}, 1 \%$ Triton X-100, 1\% sodium deoxycholate, and 0.1\% sodium dodecyl sulfate; Beyotime Co., China) containing $100 \mathrm{mM}$ phenylmethanesulfonyl fluoride (PMSF; Beyotime Co., China) for $30 \mathrm{~min}$ on ice. The lysates were centrifuged at $14,000 \times \mathrm{g}$ for $10 \mathrm{~min}$ at $4^{\circ} \mathrm{C}$, and the supernatants were collected. Twenty micrograms of protein/well was loaded onto $10 \%$ gels for separation using sodium dodecyl sulfatepolyacrylamide gels electrophoresis (SDS-PAGE). The gels were electrophoretically transferred onto polyvinylidene fluoride (PVDF) membranes $(0.45$ or $0.20 \mu \mathrm{m}$ pore size; Millipore, Billerica, MA, USA). The blotted membranes were blocked with $5 \%$ nonfat dry milk in a Tris-buffered saline solution (25 mM Tris, pH 7.5, and $150 \mathrm{mM} \mathrm{NaCl}$ ) containing 0.05\% Tween 20 (TBST) for $2 \mathrm{~h}$ at room temperature, followed by incubation with the diluted primary antibody against target protein for $4 \mathrm{~h}$ at room temperature. After washing for $10 \mathrm{~min}$ in TBST solution, the membranes were incubated with properly diluted secondary antibody conjugated with horseradish peroxidase for $2 \mathrm{~h}$ at room temperature. Western signals were developed using ECL chemiluminescent reagents from Thermo Scientific (Waltham, MA, USA). The $\beta$-actin levels were used as loading controls.

\section{Statistical analysis}

Western blotting and flow cytometry results are presented as the means \pm standard deviations (SD) from three independent experiments. Statistical analysis of the quantitative data for multiple group comparisons was performed using one-way ANOVA. Duncan's test (two sided) was used to determine the statistical significance levels $(P<0.05$ and $P<0.01)$ between control and TP-treated groups.

\section{Results}

TP-induced apoptosis of A549 cells

To investigate the effects of TP on apoptosis in A549 cells, Annexin V/PI staining-based FACS analysis was performed to detect the externalization of phosphatidylserine on the cell membrane, a hallmark of early apoptosis. Cells undergoing early-stage apoptosis were stained with Annexin V-FITC+/PI-, and late apoptotic cells were stained with Annexin V+/PI+. Fig. 1A quantifies the increase in early apoptotic cells labeled with Annexin V+/PI-, showing increases from $3.2 \%$ in the control group to $14.8,20.4 \%$ and $27.3 \%$ in the TP-treated groups, respectively. In addition, the overall apoptotic rates were significantly increased in the TP groups. These findings revealed that various TP treatments induced significantly higher 
percentages of apoptotic cells than the control group (Fig. 1B), indicating that TP can promote apoptosis.

\section{Effects of TP on cell cycle arrest}

The effects of TP on the cell cycle are shown in Fig. 2. Compared with the control group, the percentage of cells in the G0/G1 phase decreased from $63.84 \%$ to $13.75 \%$, whereas the percentage of cells in the G2/M phase increased from $14.66 \%$ to $64.56 \%$. These results indicated that TP blocks A549 cells at the G2/M phase.

Comparative proteomics of TP-treated A549 cells versus control A549 Cells

The proteins were extracted from cells treated in parallel. The samples were digested using trypsin and were labeled using 114 and 117 iTRAQ tags, and the labeled digests were utilized for MS analysis (Fig. $3 \mathrm{~A})$. The database we searched contains 376809 entries. Using ProteinPilot 4.5, we identified totals of $4977 / 5102 / 4762$ proteins in these two cell lines in the three runs (Local FDR of $<5 \%$ ). Using filters with an unused protein score of $>1.3$ and a number of peptides of $\geq 2$, $4561 / 4446 / 4302$ proteins were identified (Table 1). In total, we identified 141 up-regulated (with 117:114 iTRAQ ratios of $>2.0$ ) and 171 down-regulated proteins (with 117:114 iTRAQ ratios of $<0.5$ ) in TP-treated A549 cells compared with

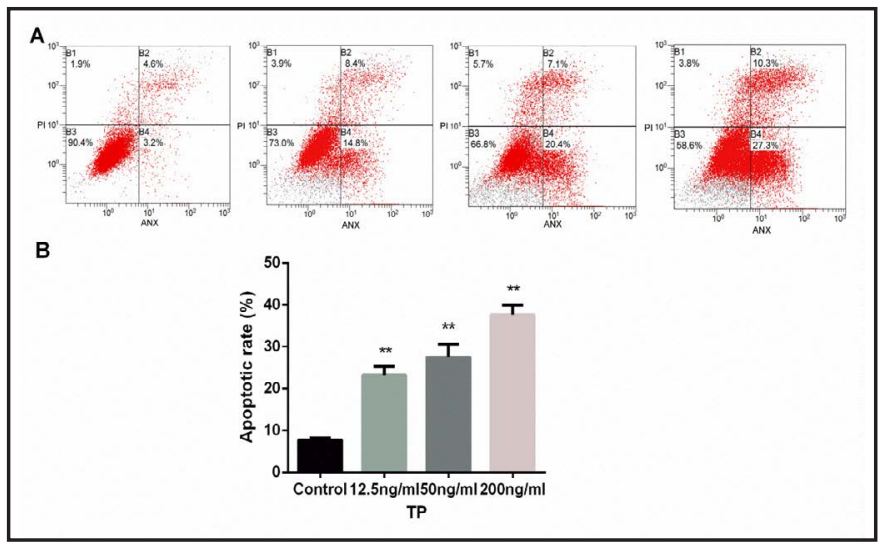

Fig. 1. Apoptotic effects of TP on human lung cancer A549 cells. (A) After $36 \mathrm{~h}$ of TP treatment (12.5, 50, and $200 \mathrm{ng} / \mathrm{ml}), \mathrm{A} 549$ cells were stained with Annexin V-FITC and PI and analyzed using flow cytometry. Early apoptotic cells were Annexin V+/ PI-, and late apoptotic cells were Annexin V+/PI+. (B) The overall apoptotic rates were significantly increased after TP treatment. ${ }^{* *} \mathrm{P}<0.01$ compared with the control group. Data are presented as the means \pm SD of three independent experiments.

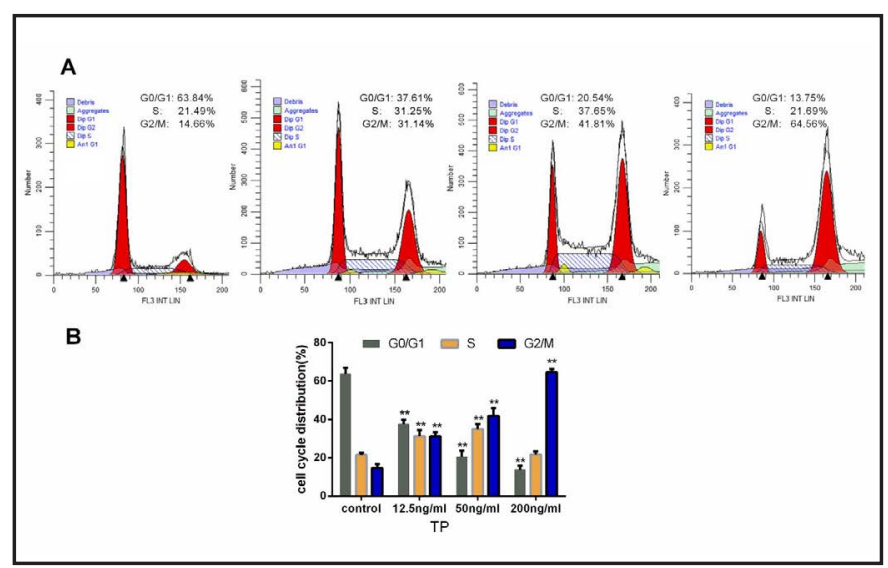

Fig. 2. Effects of TP on cell cycle distribution in human lung cancer A549 cells. (A) Representative histograms depicting cell cycle distributions in A549 cells treated with TP. After 36 $\mathrm{h}$ of TP exposure $(0,12.5,50$ and $200 \mathrm{ng} / \mathrm{ml})$, A549 cells were stained with PI and analyzed using flow cytometry. (B) The percentage of the total cell population in each phase of the cell cycle is represented as a bar diagram. ${ }^{* *} \mathrm{P}<0.01$ compared with the control group. Data are presented as the means \pm SD of three independent experiments.

Table 1. Summarized results of three iTRAQ experiments

\begin{tabular}{lcccc}
\hline $\begin{array}{l}\text { Experiment } \\
\text { No. }\end{array}$ & $\begin{array}{c}\text { Number of distinct } \\
\text { proteins } \\
\text { Local FDR }(<5 \%)\end{array}$ & $\begin{array}{c}\text { Number of distinct } \\
\text { peptides } \\
\text { Local FDR }(<5 \%)\end{array}$ & $\begin{array}{c}\text { Number of } \\
\text { spectra } \\
\text { Local FDR }(<1 \%)\end{array}$ & $\begin{array}{c}\text { Unused protein } \\
\text { score }(>1.3) \\
\text { and peptide }(>2)\end{array}$ \\
\hline 1 & 4977 & 57223 & 17567 & 4561 \\
2 & 5102 & 60205 & 17634 & 4446 \\
3 & 4762 & 56342 & 16589 & 4302 \\
\hline
\end{tabular}




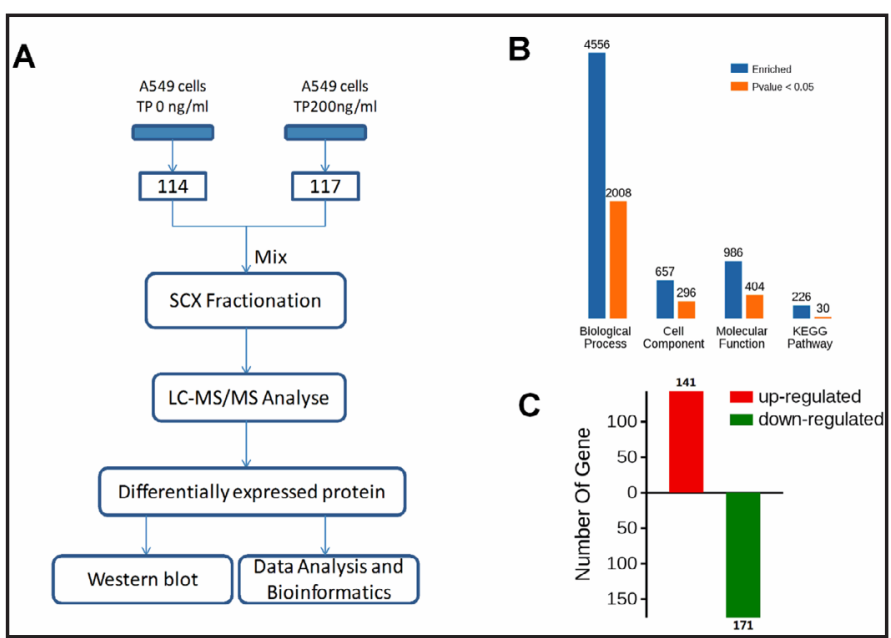

Fig. 3. Experimental quantitative proteomics analysis workflow and results. (A) Experimental design for the quantitative proteomics analysis. The proteins from A549 cells treated with different concentrations of TP were digested with trypsin and labeled using 114/117/iTRAQ tags. The labeled digests were analyzed using Nano LC-MS/MS. The differentially expressed proteins were evaluated using western blotting and analyzed through database searches. (B) The enriched counts for Biological Process, Cellular Component, Molecule Function, and KEGG Pathway. The counts for each category represent the total number of terms in the database associated with the query gene/protein list. Terms with P-values $<0.05$ are statistically significant. (C) In total, 312 proteins were identified, including 141 up-regulated proteins and 171 down-regulated proteins.

the control cells (Fig. 3C, Tables 2 and 3). Among those 312 dysregulated proteins, 50 proteins related to cell apoptosis were enriched (Table 4 ). Afterward, we performed a cluster analysis to get the heatmap which contains the data obtained for the 312 dysregulated proteins. The three horizontal clusters represent the technical replicates (Fig. 4).

\section{Functional enrichment of the TP-regulated proteins}

The obtained protein data were analyzed using bioinformatics approaches to extract information relevant to the involved pathways. Enrichments of TP-related proteins in $\mathrm{BP}, \mathrm{CC}$, and MF categories based on GO analysis are shown in Fig. 3B. In the BP analysis, the majority of identified proteins were classified into metabolic processes, particularly nitrogen compound metabolism and cellular nitrogen compound metabolism. The CC analysis showed that most of the identified proteins belonged to organelles and membrane-bounded organelles. The molecular functional classification revealed that most of these proteins were involved in binding, heterocyclic compound binding, and protein binding (Fig. 5.). GO analysis indicated that these TP-induced differentially expressed proteins exhibited a wide variety of cellular distributions and functions, consistent with the fact that TP has broad-spectrum anti-tumor effects.

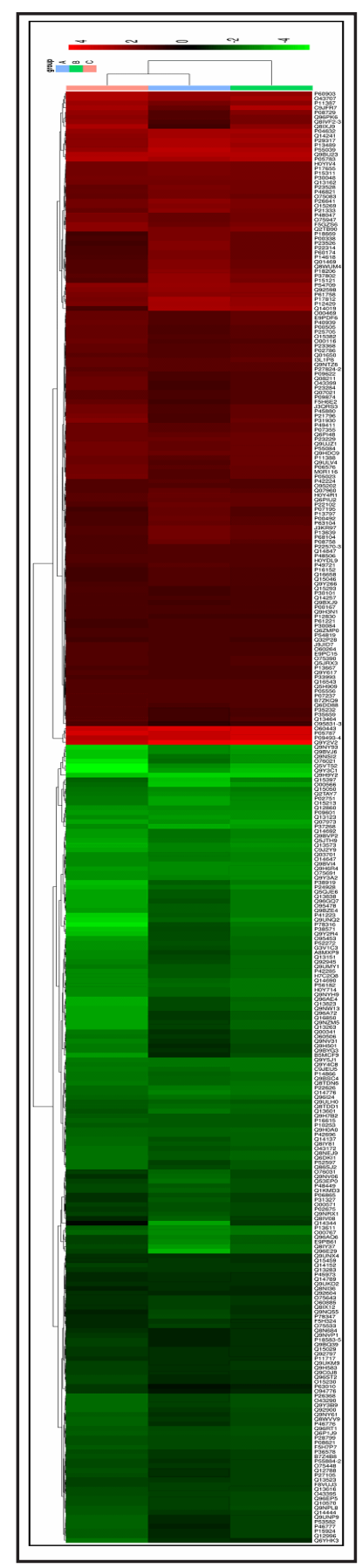

Fig. 4. Heatmap of the expression levels of 312 dysregulated proteins. The red-colored clusters represent up-regulated proteins, and the greencolored clusters represent down-regulated proteins. 
Table 2. List of up-regulated proteins in TP-treated A549 cells

\begin{tabular}{|c|c|c|c|c|}
\hline $\begin{array}{l}\text { Accession } \\
\text { No. }\end{array}$ & Gene name & Protein name & $\begin{array}{l}\text { Fold change } \\
(\text { Mean } \pm S D)\end{array}$ & $\begin{array}{c}\text { P-value } \\
(\text { Mean } \pm S D)\end{array}$ \\
\hline 095831-3 & AIFM1 & Isoform 3 of Apoptosis-inducing factor 1 & $2.05 \pm 0.04$ & $0.15 \pm 0.010$ \\
\hline P35659 & DEK & Protein DEK & $2.49 \pm 0.45$ & $0.01 \pm 0$ \\
\hline P35232 & PHB & Prohibitin & $2.61 \pm 0.4$ & $0.01 \pm 0.02$ \\
\hline Q13464 & ROCK1 & Rho-associated protein kinase 1 & $2.75 \pm 0.61$ & $0 \pm 0$ \\
\hline P30101 & PDIA3 & Protein disulfide-isomerase A3 & $2.15 \pm 0.2$ & $0 \pm 0$ \\
\hline 043399 & TPD54 & Tumor protein D54 & $2.84 \pm 0.75$ & $0.05 \pm 0$ \\
\hline Q9BXJ9 & NAA15 & $\mathrm{N}$-alpha-acetyltransferase 15 , NatA auxiliary subunit & $2.1 \pm 0.1$ & $0 \pm 0$ \\
\hline F5H6E2 & F5H6E2 & Unconventional myosin-Ic & $2.67 \pm 0.59$ & $0.01 \pm 0$ \\
\hline P23284 & PPIB & Peptidyl-prolyl cis-trans isomerase B & $2.94 \pm 0.83$ & $0 \pm 0$ \\
\hline Q15293 & RCN1 & Reticulocalbin-1 & $2.14 \pm 0.13$ & $0.02 \pm 0.01$ \\
\hline 060264 & SMCA5 & SWI/SNF-related matrix-associated actin-dependent regulator of chromatin subfamily A member 5 & $2.31 \pm 0.35$ & $0 \pm 0$ \\
\hline J9JID7 & J9JID7 & Lamin B2, isoform CRA_a & $2.39 \pm 0.36$ & $0 \pm 0$ \\
\hline Q14257 & RCN2 & Reticulocalbin-2 & $2.13 \pm 0.06$ & $0 \pm 0$ \\
\hline Q32P28 & P3H1 & Prolyl 3-hydroxylase 1 & $2.05 \pm 0.05$ & $0.02 \pm 0.02$ \\
\hline Q08211 & DHX9 & ATP-dependent RNA helicase A & $2.71 \pm 0.7$ & $0 \pm 0$ \\
\hline P09622 & DLDH & Dihydrolipoyl dehydrogenase & $2.56 \pm 0.34$ & $0.02 \pm 0$ \\
\hline E9PC15 & E9PC15 & Acylglycerol kinase & $2.28 \pm 0.24$ & $0 \pm 0$ \\
\hline P09874 & PARP1 & Poly [ADP-ribose] polymerase 1 & $2.48 \pm 0.46$ & $0 \pm 0.01$ \\
\hline Q5JRX3 & PREP & Presequence protease & $2.39 \pm 0.34$ & $0 \pm 0$ \\
\hline Q9H3N1 & TMX1 & Thioredoxin-related transmembrane protein 1 & $2.19 \pm 0.06$ & $0.02 \pm 0$ \\
\hline P00167 & CYB5 & Cytochrome b5 & $2.2 \pm 0.07$ & $0.04 \pm 0.01$ \\
\hline P54819 & KAD2 & Adenylate kinase 2 & $2.12 \pm 0.07$ & $0.01 \pm 0.02$ \\
\hline 075390 & CISY & Citrate synthase & $2.41 \pm 0.31$ & $0 \pm 0$ \\
\hline P21796 & VDAC1 & Voltage-dependent anion-selective channel protein 1 & $3.01 \pm 0.17$ & $0 \pm 0$ \\
\hline P30084 & ECHM & Enoyl-CoA hydratase & $2.11 \pm 0.01$ & $0.02 \pm 0.01$ \\
\hline Q07021 & $\mathrm{C} 1 \mathrm{QBP}$ & Complement component $1 \mathrm{Q}$ subcomponent-binding protein & $2.52 \pm 0.47$ & $0.03 \pm 0.01$ \\
\hline Q6ZMP0 & THSD 4 & Thrombospondin type-1 domain-containing protein 4 & $2.14 \pm 0.07$ & $0.04 \pm 0$ \\
\hline 000116 & ADAS & Alkyldihydroxyacetonephosphate synthase, peroxisomal & $2.65 \pm 0.6$ & $0.03 \pm 0.01$ \\
\hline P45880 & VDAC2 & Voltage-dependent anion-selective channel protein 2 & $2.93 \pm 0.69$ & $0 \pm 0$ \\
\hline Q9Y617 & SERC & Phosphoserine aminotransferase & $2.31 \pm 0.09$ & $0 \pm 0$ \\
\hline P61221 & ABCE1 & ATP-binding cassette sub-family E member 1 & $2.14 \pm 0.15$ & $0 \pm 0$ \\
\hline J3QRS3 & J3QRS3 & Myosin regulatory light chain $12 \mathrm{~A}$ & $2.96 \pm 1$ & $0.01 \pm 0.02$ \\
\hline P13667 & PDIA4 & Protein disulfide-isomerase A4 & $2.3 \pm 0.04$ & $0 \pm 0$ \\
\hline P25705 & ATPA & ATP synthase subunit alpha & $2.76 \pm 0.36$ & $0 \pm 0$ \\
\hline P00505 & AATM & Aspartate aminotransferase & $2.74 \pm 0.64$ & $0 \pm 0$ \\
\hline P33993 & MCM7 & DNA replication licensing factor MCM7 & $2.26 \pm 0.04$ & $0.03 \pm 0.01$ \\
\hline Q6DD88 & ATLA3 & Atlastin-3 & $2.49 \pm 0.27$ & $0 \pm 0$ \\
\hline Q9Y266 & NUDC & Nuclear migration protein nudC & $2.21 \pm 0.2$ & $0 \pm 0$ \\
\hline B7ZKQ9 & B7ZKQ9 & SCARB1 protein & $2.53 \pm 0.25$ & $0.03 \pm 0.01$ \\
\hline P07237 & PDIA1 & Protein disulfide-isomerase & $2.54 \pm 0.23$ & $0 \pm 0$ \\
\hline P05556 & ITB1 & Integrin beta- 1 & $2.46 \pm 0.11$ & $0 \pm 0$ \\
\hline Q15046 & SYK & Lysine--tRNA ligase & $2.8 \pm 0.26$ & $0.01 \pm 0.02$ \\
\hline 015382 & BCAT2 & Branched-chain-amino-acid aminotransferase & $2.77 \pm 0.56$ & $0.02 \pm 0.01$ \\
\hline Q14847 & LASP1 & LIM and SH3 domain protein 1 & $2.35 \pm 0.11$ & $0 \pm 0$ \\
\hline Q5H909 & Q5H909 & Melanoma-associated antigen D2 & $2.51 \pm 0.13$ & $0.02 \pm 0.01$ \\
\hline P40939 & ECHA & Trifunctional enzyme subunit alpha & $2.97 \pm 0.71$ & $0 \pm 0$ \\
\hline Q16543 & CDC37 & Hsp90 co-chaperone Cdc37 & $2.52 \pm 0.08$ & $0 \pm 0$ \\
\hline Q16658 & FSCN1 & Fascin & $2.25 \pm 0.34$ & $0 \pm 0$ \\
\hline HOYDL9 & HOYDL9 & CD81 antigen (Fragment) & $2.44 \pm 0.06$ & $0.04 \pm 0$ \\
\hline E9PDF6 & E9PDF6 & Unconventional myosin-Ib & $2.88 \pm 0.52$ & $0 \pm 0$ \\
\hline P05023 & AT1A1 & Sodium/potassium-transporting ATPase subunit alpha-1 & $3.17 \pm 0.94$ & $0 \pm 0$ \\
\hline P02786 & TFR1 & Transferrin receptor protein 1 & $2.72 \pm 0.3$ & $0 \pm 0$ \\
\hline P48506 & GSH1 & Glutamate--cysteine ligase catalytic subunit & $2.49 \pm 0.03$ & $0.01 \pm 0.01$ \\
\hline P22570-3 & ADRO & Isoform 3 of NADPH: adrenodoxin oxidoreductase & $2.42 \pm 0.16$ & $0 \pm 0$ \\
\hline Q01650 & LAT1 & Large neutral amino acids transporter small subunit 1 & $2.68 \pm 0.17$ & $0.01 \pm 0.02$ \\
\hline 000469 & PLOD2 & Procollagen-lysine,2-oxoglutarate 5-dioxygenase 2 & $2.86 \pm 0.39$ & $0 \pm 0$ \\
\hline P16152 & CBR1 & Carbonyl reductase [NADPH] 1 & $2.31 \pm 0.39$ & $0 \pm 0$ \\
\hline 095202 & LETM1 & LETM1 and EF-hand domain-containing protein 1 & $2.61 \pm 0.03$ & $0 \pm 0$ \\
\hline P23368 & MAOM & NAD-dependent malic enzyme & $2.78 \pm 0.22$ & $0.03 \pm 0.01$ \\
\hline Q9ULV4 & COR1C & Coronin-1C & $3.06 \pm 0.57$ & $0 \pm 0$ \\
\hline M0R116 & M0R116 & Sodium/potassium-transporting ATPase subunit alpha- 3 & $3.13 \pm 0.67$ & $0.05 \pm 0$ \\
\hline P42224 & STAT1 & Signal transducer and activator of transcription 1-alpha/beta & $2.62 \pm 0.09$ & $0 \pm 0$ \\
\hline P49721 & PSB2 & Proteasome subunit beta type- 2 & $2.41 \pm 0.38$ & $0.03 \pm 0.02$ \\
\hline P11388 & TOP2A & DNA topoisomerase 2-alpha & $3.07 \pm 0.52$ & $0 \pm 0$ \\
\hline P06576 & ATPB & ATP synthase subunit beta & $3.13 \pm 0.53$ & $0 \pm 0$ \\
\hline Q9NTZ6 & RBM12 & RNA-binding protein 12 & $2.85 \pm 0.13$ & $0.01 \pm 0.01$ \\
\hline P12830 & $\mathrm{CDH} 1$ & Cadherin-1 & $2.30 \pm 0.10$ & $0.04 \pm 0.01$ \\
\hline P07355 & ANXA2 & Annexin A2 & $2.94 \pm 0.23$ & $0 \pm 0$ \\
\hline P27824-2 & CALX & Isoform 2 of Calnexin & $2.81 \pm 0.04$ & $0 \pm 0$ \\
\hline P49411 & EFTU & Elongation factor $\mathrm{Tu}$ & $3 \pm 0.31$ & $0 \pm 0$ \\
\hline Q96PK6 & RBM14 & RNA-binding protein 14 & $3.89 \pm 0.53$ & $0.01 \pm 0.01$ \\
\hline I3L1P8 & I3L1P8 & Mitochondrial 2-oxoglutarate/malate carrier protein (Fragment) & $2.89 \pm 0.11$ & $0 \pm 0$ \\
\hline P55084 & ECHB & Trifunctional enzyme subunit beta & $3.27 \pm 0.51$ & $0.01 \pm 0.02$ \\
\hline Q6PI48 & SYDM & Aspartate--tRNA ligase & $3.07 \pm 0.22$ & $0 \pm 0$ \\
\hline
\end{tabular}




\begin{tabular}{|c|c|c|c|c|}
\hline $\begin{array}{c}\text { Accession } \\
\text { No. }\end{array}$ & Gene name & Protein name & $\begin{array}{l}\text { Fold change } \\
(\text { Mean } \pm \text { SD) }\end{array}$ & $\begin{array}{c}\text { P-value } \\
(\text { Mean } \pm S D)\end{array}$ \\
\hline Q6PIU2 & NCEH1 & Neutral cholesterol ester hydrolase 1 & $2.9 \pm 0.13$ & $0 \pm 0$ \\
\hline Q07960 & RHG01 & Rho GTPase-activating protein 1 & $2.72 \pm 0.39$ & $0.01 \pm 0$ \\
\hline P13797 & PLST & Plastin-3 & $2.61 \pm 0.66$ & $0 \pm 0$ \\
\hline P31930 & QCR1 & Cytochrome b-c1 complex subunit 1 & $3.18 \pm 0.14$ & $0 \pm 0$ \\
\hline H0Y4R1 & H0Y4R1 & Inosine-5'-monophosphate dehydrogenase 2 (Fragment) & $2.9 \pm 0.28$ & $0 \pm 0$ \\
\hline P07195 & LDHB & L-lactate dehydrogenase B chain & $2.59 \pm 0.77$ & $0 \pm 0$ \\
\hline 075947 & ATP5H & ATP synthase subunit $d$ & $3.63 \pm 0.71$ & $0.02 \pm 0.01$ \\
\hline P48047 & ATPO & ATP synthase subunit 0 & $3.65 \pm 0.73$ & $0.04 \pm 0.01$ \\
\hline Q9HDC9 & APMAP & Adipocyte plasma membrane-associated protein & $3.39 \pm 0.29$ & $0 \pm 0$ \\
\hline P23229 & ITA6 & Integrin alpha-6 & $3.16 \pm 0.08$ & $0 \pm 0$ \\
\hline P63104 & $1433 Z$ & 14-3-3 protein zeta/delta & $2.71 \pm 0.77$ & $0 \pm 0$ \\
\hline 043707 & ACTN4 & Alpha-actinin-4 & $6.61 \pm 1.84$ & $0 \pm 0$ \\
\hline Q8IVF2-3 & AHNK2 & Isoform 3 of Protein AHNAK2 & $3.94 \pm 0.84$ & $0 \pm 0$ \\
\hline P00492 & HPRT & Hypoxanthine-guanine phosphoribosyltransferase & $2.75 \pm 0.83$ & $0 \pm 0$ \\
\hline F5GZS6 & F5GZS6 & $4 \mathrm{~F} 2$ cell-surface antigen heavy chain & $4.01 \pm 0.91$ & $0 \pm 0$ \\
\hline P22102 & PUR2 & Trifunctional purine biosynthetic protein adenosine- 3 & $2.99 \pm 0.54$ & $0 \pm 0$ \\
\hline P08758 & ANXA5 & Annexin A5 & $2.85 \pm 0.73$ & $0 \pm 0$ \\
\hline Q13162 & PRDX4 & Peroxiredoxin- 4 & $3.56 \pm 0.05$ & $0.01 \pm 0.02$ \\
\hline P13639 & EF2 & Elongation factor 2 & $2.98 \pm 0.38$ & $0 \pm 0$ \\
\hline J3KR97 & J3 KR97 & Tubulin-specific chaperone D & $2.99 \pm 0.16$ & $0.01 \pm 0.01$ \\
\hline P68104 & EF1A1 & Elongation factor 1 -alpha 1 & $2.98 \pm 0.97$ & $0 \pm 0$ \\
\hline 075083 & WDR1 & WD repeat-containing protein 1 & $3.35 \pm 0.54$ & $0 \pm 0$ \\
\hline P08729 & $\mathrm{K} 2 \mathrm{C} 7$ & Keratin, type II cytoskeletal 7 & $4.32 \pm 0.78$ & $0 \pm 0$ \\
\hline P46821 & MAP1B & Microtubule-associated protein $1 \mathrm{~B}$ & $3.5 \pm 0.43$ & $0 \pm 0$ \\
\hline P11387 & TOP1 & DNA topoisomerase 1 & $5.03 \pm 1.64$ & $0 \pm 0$ \\
\hline P30048 & PRDX3 & Thioredoxin-dependent peroxide reductase & $3.85 \pm 0.03$ & $0.02 \pm 0.01$ \\
\hline P15311 & EZRI & Ezrin & $3.79 \pm 0.27$ & $0 \pm 0$ \\
\hline P14618 & KPYM & Pyruvate kinase PKM & $3.18 \pm 1.23$ & $0 \pm 0$ \\
\hline Q2TB90 & HKDC1 & Putative hexokinase HKDC1 & $4.31 \pm 0.36$ & $0 \pm 0$ \\
\hline P23528 & COF1 & Cofilin-1 & $3.55 \pm 0.71$ & $0 \pm 0$ \\
\hline Q8IXJ9 & ASXL1 & Putative Polycomb group protein ASXL1 & $4.85 \pm 1.13$ & $0.04 \pm 0.01$ \\
\hline P00338 & LDHA & L-lactate dehydrogenase A chain & $3.09 \pm 1.42$ & $0 \pm 0$ \\
\hline P17655 & CAN2 & Calpain- 2 catalytic subunit & $3.81 \pm 0.4$ & $0 \pm 0$ \\
\hline P18669 & PGAM1 & Phosphoglycerate mutase 1 & $3.08 \pm 1.48$ & $0 \pm 0$ \\
\hline P60174 & TPIS & Triosephosphate isomerase & $3.17 \pm 1.41$ & $0 \pm 0$ \\
\hline HOYIV4 & H0YIV4 & Nucleosome assembly protein 1-like 1 (Fragment) & $3.85 \pm 0.45$ & $0 \pm 0$ \\
\hline P22314 & UBA1 & Ubiquitin-like modifier-activating enzyme 1 & $3.19 \pm 1.5$ & $0 \pm 0$ \\
\hline P23526 & SAHH & Adenosylhomocysteinase & $3.15 \pm 1.55$ & $0 \pm 0$ \\
\hline Q8WUM4 & PDC6I & Programmed cell death 6-interacting protein & $3.43 \pm 1.2$ & $0 \pm 0$ \\
\hline Q01469 & FABP5 & Fatty acid-binding protein, epidermal & $3.41 \pm 1.24$ & $0.01 \pm 0.01$ \\
\hline 015269 & SPTC1 & Serine palmitoyltransferase 1 & $4.05 \pm 0.5$ & $0.02 \pm 0.01$ \\
\hline P21333 & FLNA & Filamin-A & $3.92 \pm 1.11$ & $0 \pm 0$ \\
\hline P18206 & VINC & Vinculin & $3.62 \pm 1.53$ & $0 \pm 0$ \\
\hline P26641 & EF1G & Elongation factor 1 -gamma & $4.23 \pm 0.98$ & $0 \pm 0$ \\
\hline P61758 & PFD3 & Prefoldin subunit 3 & $4.59 \pm 0.6$ & $0 \pm 0$ \\
\hline C9JFR7 & C9JFR7 & Cytochrome c (Fragment) & $5.99 \pm 1.32$ & $0 \pm 0$ \\
\hline P15121 & ALDR & Aldose reductase & $3.84 \pm 1.92$ & $0 \pm 0$ \\
\hline Q92598 & HS105 & Heat shock protein $105 \mathrm{kDa}$ & $4.88 \pm 0.73$ & $0 \pm 0$ \\
\hline P37802 & TAGL2 & Transgelin-2 & $4.12 \pm 1.94$ & $0 \pm 0$ \\
\hline $\mathrm{P} 60903$ & S10AA & Protein S100-A10 & $6.33 \pm 1.11$ & $0.04 \pm 0.01$ \\
\hline P54709 & AT1B3 & Sodium/potassium-transporting ATPase subunit beta-3 & $5.15 \pm 0.7$ & $0.01 \pm 0.01$ \\
\hline P12429 & ANXA3 & Annexin A3 & $5.26 \pm 1.65$ & $0 \pm 0$ \\
\hline P17812 & PYRG1 & CTP synthase 1 & $5.27 \pm 2.07$ & $0 \pm 0$ \\
\hline Q14241 & ELOA1 & Transcription elongation factor B polypeptide 3 & $6.04 \pm 1.33$ & $0.04 \pm 0.01$ \\
\hline Q14019 & COTL1 & Coactosin-like protein & $5.08 \pm 2.79$ & $0 \pm 0$ \\
\hline P05783 & K1C18 & Keratin, type I cytoskeletal 18 & $7.87 \pm 1.07$ & $0 \pm 0$ \\
\hline P04632 & CPNS1 & Calpain small subunit 1 & $6.43 \pm 0.96$ & $0.03 \pm 0.01$ \\
\hline P13489 & RINI & Ribonuclease inhibitor & $6.26 \pm 2.27$ & $0.01 \pm 0.01$ \\
\hline P29317 & EPHA2 & Ephrin type-A receptor 2 & $6.39 \pm 2.2$ & $0 \pm 0$ \\
\hline Q9BU23 & LMF2 & Lipase maturation factor 2 & $8.28 \pm 0.27$ & $0 \pm 0$ \\
\hline P55039 & DRG2 & Developmentally regulated GTP-binding protein 2 & $7.22 \pm 2.22$ & $0.04 \pm 0$ \\
\hline P09493-4 & TPM1 & Isoform 4 of Tropomyosin alpha- 1 chain & $10.42 \pm 0.2$ & $0.02 \pm 0.01$ \\
\hline P05787 & $\mathrm{K} 2 \mathrm{C} 8$ & Keratin, type II cytoskeletal 8 & $11.78 \pm 1.3$ & $0 \pm 0$ \\
\hline 060443 & DFNA5 & Non-syndromic hearing impairment protein 5 & $11.92 \pm 1.78$ & $0.03 \pm 0.01$ \\
\hline Q9Y2V2 & CHSP1 & Calcium-regulated heat stable protein 1 & $16.91 \pm 2.3$ & $0.03 \pm 0.02$ \\
\hline
\end{tabular}

\section{KEGG pathway analysis}

KEGG analysis revealed 30 significant pathways with $P<0.05$ (Fig. 3B.). The top ten pathways, including Ribosome biogenesis in eukaryotes (hsa03008), Spliceosome (hsa03040), mRNA surveillance pathway (hsa03015), Carbon metabolism (hsa01200), Steroid biosynthesis (hsa00100), Cysteine and methionine metabolism (hsa00270), Propanoate metabolism (hsa00640), Glycolysis/Gluconeogenesis (hsa00010), Biosynthesis of amino acids (hsa01230), and Cardiac muscle contraction (hsa04260), were displayed. Ribosome biogenesis in eukaryotes and spliceosome were the most significantly enriched pathways (Fig. 6). 
Table 3. List of down-regulated proteins in TP-treated A549 cells

\begin{tabular}{|c|c|c|c|c|}
\hline $\begin{array}{c}\text { Accession } \\
\text { NO. }\end{array}$ & Gene name & Protein name & $\begin{array}{l}\text { Fold change } \\
(\text { Mean } \pm S D)\end{array}$ & $\begin{array}{c}\text { P-value } \\
(\text { Mean } \pm S D)\end{array}$ \\
\hline Q9H9Y2 & RPF1 & Ribosome production factor 1 & $0.06 \pm 0.01$ & $0.02 \pm 0.01$ \\
\hline P02675 & FIBB & Fibrinogen beta chain & $0.32 \pm 0.07$ & $0 \pm 0$ \\
\hline Q96E29 & MTEF3 & Transcription termination factor 3 & $0.21 \pm 0.19$ & $0.02 \pm 0.01$ \\
\hline P37268 & FDFT & Squalene synthase & $0.11 \pm 0.04$ & $0 \pm 0$ \\
\hline 015213 & WDR46 & WD repeat-containing protein 46 & $0.13 \pm 0.04$ & $0 \pm 0$ \\
\hline P02751 & FINC & Fibronectin & $0.14 \pm 0.05$ & $0 \pm 0$ \\
\hline 000566 & MPP10 & U3 small nucleolar ribonucleoprotein protein MPP10 & $0.11 \pm 0$ & $0 \pm 0$ \\
\hline Q8IY37 & DHX37 & Probable ATP-dependent RNA helicase DHX37 & $0.21 \pm 0.15$ & $0 \pm 0$ \\
\hline Q13123 & RED & Protein Red & $0.12 \pm 0.01$ & $0 \pm 0$ \\
\hline Q9Y3C1 & NOP16 & Nucleolar protein 16 & $0.07 \pm 0.06$ & $0.01 \pm 0.01$ \\
\hline Q07973 & CP24A & 1,25-dihydroxyvitamin $D(3)$ 24-hydroxylase & $0.1 \pm 0.02$ & $0 \pm 0$ \\
\hline Q15397 & K0020 & Pumilio domain-containing protein KIAA0020 & $0.15 \pm 0.05$ & $0 \pm 0$ \\
\hline Q2TAY7 & SMU1 & WD40 repeat-containing protein SMU1 & $0.15 \pm 0.04$ & $0 \pm 0$ \\
\hline P09601 & HMOX1 & Heme oxygenase 1 & $0.12 \pm 0.01$ & $0 \pm 0$ \\
\hline 000767 & ACOD & Acyl-CoA desaturase & $0.17 \pm 0.06$ & $0 \pm 0.01$ \\
\hline Е9PB61 & E9PB61 & THO complex subunit 4 & $0.24 \pm 0.16$ & $0 \pm 0$ \\
\hline Q15050 & RRS1 & Ribosome biogenesis regulatory protein homolog & $0.16 \pm 0.04$ & $0 \pm 0$ \\
\hline Q12860 & CNTN1 & Contactin-1 & $0.13 \pm 0.01$ & $0 \pm 0$ \\
\hline Q5VT52 & RPRD2 & Regulation of nuclear pre-mRNA domain-containing protein 2 & $0.08 \pm 0.07$ & $0.03 \pm 0.02$ \\
\hline P13611 & CSPG2 & Versican core protein & $0.19 \pm 0.07$ & $0.01 \pm 0.01$ \\
\hline Q9BVJ6 & UT14A & U3 small nucleolar RNA-associated protein 14 homolog A & $0.11 \pm 0.06$ & $0 \pm 0$ \\
\hline Q9BVI4 & NOC4L & Nucleolar complex protein 4 homolog & $0.14 \pm 0$ & $0 \pm 0$ \\
\hline 075691 & UTP20 & Small subunit processome component 20 homolog & $0.13 \pm 0.02$ & $0 \pm 0$ \\
\hline Q9H6R4 & NOL6 & Nucleolar protein 6 & $0.13 \pm 0.03$ & $0 \pm 0$ \\
\hline Q9NY93 & DDX56 & Probable ATP-dependent RNA helicase DDX56 & $0.1 \pm 0.07$ & $0 \pm 0$ \\
\hline 000571 & DDX3X & ATP-dependent RNA helicase DDX3X & $0.32 \pm 0.07$ & $0.02 \pm 0.02$ \\
\hline Q96AQ6 & PBIP1 & Pre-B-cell leukemia transcription factor-interacting protein 1 & $0.24 \pm 0.13$ & $0 \pm 0$ \\
\hline Q9Y3A2 & UTP11 & Probable U3 small nucleolar RNA-associated protein 11 & $0.14 \pm 0.03$ & $0.04 \pm 0.01$ \\
\hline С9J2Y9 & C9J2Y9 & DNA-directed RNA polymerase & $0.13 \pm 0.04$ & $0 \pm 0$ \\
\hline Q13573 & SNW1 & SNW domain-containing protein 1 & $0.12 \pm 0.05$ & $0.03 \pm 0.01$ \\
\hline 014776 & TCRG1 & Transcription elongation regulator 1 & $0.18 \pm 0.03$ & $0 \pm 0$ \\
\hline P22626 & ROA2 & Heterogeneous nuclear ribonucleoproteins A2/B1 & $0.18 \pm 0.03$ & $0 \pm 0$ \\
\hline 014647 & CHD2 & Chromodomain-helicase-DNA-binding protein 2 & $0.15 \pm 0.03$ & $0.04 \pm 0.01$ \\
\hline Q03701 & CEBPZ & CCAAT/enhancer-binding protein zeta & $0.15 \pm 0.03$ & $0 \pm 0$ \\
\hline Q9Y4C8 & RBM19 & Probable RNA-binding protein 19 & $0.17 \pm 0$ & $0.02 \pm 0.02$ \\
\hline Q5JTH9 & RRP12 & RRP12-like protein & $0.14 \pm 0.05$ & $0 \pm 0$ \\
\hline Q9Y5J1 & UTP18 & U3 small nucleolar RNA-associated protein 18 homolog & $0.17 \pm 0.01$ & $0 \pm 0$ \\
\hline Q13601 & KRR1 & KRR1 small subunit processome component homolog & $0.23 \pm 0.07$ & $0 \pm 0$ \\
\hline Q9NSI2 & F207A & Protein FAM207A & $0.12 \pm 0.08$ & $0.01 \pm 0.01$ \\
\hline Q9BVP2 & GNL3 & Guanine nucleotide-binding protein-like 3 & $0.15 \pm 0.05$ & $0.02 \pm 0.01$ \\
\hline Q53EP0 & FND3B & Fibronectin type III domain-containing protein 3B & $0.27 \pm 0.11$ & $0 \pm 0$ \\
\hline Q14692 & BMS1 & Ribosome biogenesis protein BMS1 homolog & $0.15 \pm 0.06$ & $0 \pm 0$ \\
\hline Q96I24 & FUBP3 & Far upstream element-binding protein 3 & $0.2 \pm 0.01$ & $0 \pm 0$ \\
\hline P24928 & RPB1 & DNA-directed RNA polymerase II subunit RPB1 & $0.14 \pm 0.08$ & $0 \pm 0$ \\
\hline P56182 & RRP1 & Ribosomal RNA processing protein 1 homolog A & $0.17 \pm 0.04$ & $0 \pm 0$ \\
\hline Q9NV06 & DCA13 & DDB1- and CUL4-associated factor 13 & $0.3 \pm 0.15$ & $0 \pm 0$ \\
\hline 076021 & RL1D1 & Ribosomal L1 domain-containing protein 1 & $0.12 \pm 0.11$ & $0 \pm 0$ \\
\hline C9JEU5 & C9JEU5 & Fibrinogen gamma chain & $0.18 \pm 0.03$ & $0 \pm 0$ \\
\hline Q8TDD1 & DDX54 & ATP-dependent RNA helicase DDX54 & $0.22 \pm 0.02$ & $0 \pm 0$ \\
\hline Q14690 & RRP5 & Protein RRP5 homolog & $0.17 \pm 0.05$ & $0 \pm 0$ \\
\hline 076031 & CLPX & ATP-dependent Clp protease ATP-binding subunit clpX-like & $0.29 \pm 0.1$ & $0 \pm 0$ \\
\hline Q9BSC4 & NOL10 & Nucleolar protein 10 & $0.2 \pm 0.03$ & $0 \pm 0$ \\
\hline Q8TDN6 & BRX1 & Ribosome biogenesis protein BRX1 homolog & $0.19 \pm 0.04$ & $0.01 \pm 0.01$ \\
\hline Q9ULH0 & KDIS & Kinase D-interacting substrate of $220 \mathrm{kDa}$ & $0.24 \pm 0.03$ & $0 \pm 0$ \\
\hline Q9NYH9 & UTP6 & U3 small nucleolar RNA-associated protein 6 homolog & $0.17 \pm 0.07$ & $0.02 \pm 0.02$ \\
\hline H0Y714 & H0Y714 & U3 small nucleolar ribonucleoprotein protein IMP4 (Fragment) & $0.18 \pm 0.07$ & $\begin{array}{l}0 \pm 0 \\
0 \pm 0\end{array}$ \\
\hline P14866 & HNRPL & Heterogeneous nuclear ribonucleoprotein L & $0.2 \pm 0.04$ & \\
\hline Q9UNQ2 & DIM1 & Probable dimethyladenosine transferase & $0.14 \pm 0.13$ & $0.04 \pm 0$ \\
\hline Q1KMD3 & HNRL2 & Heterogeneous nuclear ribonu cleoprotein U-like protein 2 & $0.28 \pm 0.06$ & $0 \pm 0$ \\
\hline P42696 & RBM34 & RNA-binding protein 34 & $0.23 \pm 0.01$ & $0.01 \pm 0.02$ \\
\hline Q9H0A0 & NAT10 & $\mathrm{N}$-acetyltransferase 10 & $0.22 \pm 0.02$ & $0 \pm 0$ \\
\hline Q8IV08 & PLD3 & Phospholipase D3 & $0.35 \pm 0.16$ & $0.01 \pm 0.02$ \\
\hline Q9BZE4 & NOG1 & Nucleolar GTP-binding protein 1 & $0.17 \pm 0.09$ & $0 \pm 0$ \\
\hline P38919 & EIF4A3 & Eukaryotic initiation factor 4A-III & $0.16 \pm 0.12$ & $0 \pm 0$ \\
\hline 095478 & NSA2 & Ribosome biogenesis protein NSA2 homolog & $0.17 \pm 0.1$ & $0 \pm 0$ \\
\hline P48449 & ERG7 & Lanosterol synthase & $0.28 \pm 0.04$ & $0 \pm 0$ \\
\hline P31327 & CPSM & Carbamoyl-phosphate synthase [ammonia] & $0.33 \pm 0.11$ & $0 \pm 0$ \\
\hline P16615 & AT2A2 & Sarcoplasmic/endoplasmic reticulum calcium ATPase 2 & $0.24 \pm 0.01$ & $0 \pm 0$ \\
\hline P10253 & LYAG & Lysosomal alpha-glucosidase & $0.25 \pm 0$ & $0.01 \pm 0.01$ \\
\hline Q6DKI1 & RL7L & $60 \mathrm{~S}$ ribosomal protein L7-like 1 & $0.22 \pm 0.05$ & $0.04 \pm 0.01$ \\
\hline Q9H7B2 & RPF2 & Ribosome production factor 2 homolog & $0.24 \pm 0.01$ & $0.05 \pm 0$ \\
\hline Q8NEJ9 & NGDN & Neuroguidin & $0.22 \pm 0.05$ & $0 \pm 0$ \\
\hline Q13838 & DX39B & Spliceosome RNA helicase DDX39B & $0.17 \pm 0.12$ & $0.02 \pm 0.02$ \\
\hline Q5QJE6 & TDIF2 & Deoxynucleotidyltransferase terminal-interacting protein 2 & $0.17 \pm 0.12$ & $0.04 \pm 0.01$ \\
\hline $\mathrm{H} 7 \mathrm{C} 2 \mathrm{Q} 8$ & H7C2Q8 & EBNA1 binding protein 2, isoform CRA_d & $0.2 \pm 0.08$ & $0 \pm 0$ \\
\hline P41223 & BUD31 & Protein BUD31 homolog & $0.16 \pm 0.14$ & $0.03 \pm 0.01$ \\
\hline Q9NRX1 & PNO1 & RNA-binding protein PNO1 & $0.32 \pm 0.08$ & $0 \pm 0$ \\
\hline P42285 & SK2L2 & Superkiller viralicidic activity 2 -like 2 & $0.2 \pm 0.09$ & $0 \pm 0$ \\
\hline Q9UMY1 & NOL7 & Nucleolar protein 7 & $0.21 \pm 0.07$ & $0 \pm 0$ \\
\hline Q8IY81 & SPB1 & pre-rRNA processing protein FTSJ3 & $0.23 \pm 0.05$ & $0 \pm 0$ \\
\hline Q14137 & BOP1 & Ribosome biogenesis protein $\mathrm{BOP} 1$ & $0.23 \pm 0.05$ & $0 \pm 0$ \\
\hline Q96GQ7 & DDX27 & Probable ATP-dependent RNA helicase DDX27 & $0.18 \pm 0.13$ & $0 \pm 0$ \\
\hline P06865 & HEXA & Beta-hexosaminidase subunit alpha & $0.34 \pm 0.09$ & $0 \pm 0$ \\
\hline P52272 & HNRPM & Heterogeneous nuclear ribonucleoprotein $\mathrm{M}$ & $0.2 \pm 0.11$ & $0 \pm 0$ \\
\hline
\end{tabular}




\begin{tabular}{|c|c|c|c|c|}
\hline $\begin{array}{c}\text { Accession } \\
\text { NO. }\end{array}$ & Gene name & Protein name & $\begin{array}{l}\text { Fold change } \\
(\text { Mean } \pm S D)\end{array}$ & $\begin{array}{c}\text { P-value } \\
(\text { Mean } \pm S D)\end{array}$ \\
\hline 043172 & PRP4 & U4/U6 small nuclear ribonucleoprotein Prp4 & $0.24 \pm 0.07$ & $0 \pm 0$ \\
\hline Q92945 & FUBP2 & Far upstream element-binding protein 2 & $0.22 \pm 0.1$ & $0 \pm 0$ \\
\hline 095453 & PARN & Poly(A)-specific ribonuclease PARN & $0.2 \pm 0.12$ & $0 \pm 0$ \\
\hline Q6P1J9 & CDC73 & Parafibromin & $0.27 \pm 0.03$ & $0 \pm 0$ \\
\hline Q14344 & GNA13 & Guanine nucleotide-binding protein subunit alpha-13 & $0.39 \pm 0.12$ & $0.01 \pm 0.01$ \\
\hline P78316 & NOP14 & Nucleolar protein 14 & $0.17 \pm 0.18$ & $0 \pm 0$ \\
\hline Q9Y2R4 & DDX52 & Probable ATP-dependent RNA helicase DDX52 & $0.19 \pm 0.16$ & $0.01 \pm 0.01$ \\
\hline P78347 & GTF2I & General transcription factor II-I & $0.38 \pm 0.11$ & $0 \pm 0$ \\
\hline G3V1C3 & G3V1C3 & Apoptosis inhibitor 5 & $0.21 \pm 0.13$ & $0 \pm 0$ \\
\hline Q9NW13 & RBM28 & RNA-binding protein 28 & $0.2 \pm 0.15$ & $0 \pm 0$ \\
\hline Q86SJ2 & AMGO2 & Amphoterin-induced protein 2 & $0.25 \pm 0.08$ & $0 \pm 0$ \\
\hline Q13823 & NOG2 & Nucleolar GTP-binding protein 2 & $0.2 \pm 0.16$ & $0 \pm 0$ \\
\hline Q13151 & ROA0 & Heterogeneous nuclear ribonucleoprotein $\mathrm{A} 0$ & $0.22 \pm 0.13$ & $0.04 \pm 0.01$ \\
\hline Q96RT1 & LAP2 & Protein LAP2 & $0.28 \pm 0.04$ & $0.01 \pm 0.01$ \\
\hline P08621 & RU17 & U1 small nuclear ribonucleoprotein $70 \mathrm{kDa}$ & $0.3 \pm 0.03$ & $0 \pm 0$ \\
\hline Q9NQ55 & SSF1 & Suppressor of SWI4 1 homolog & $0.4 \pm 0.12$ & $0.01 \pm 0.01$ \\
\hline F5H7P7 & F5H7P7 & Peptidylprolyl isomerase domain and WD repeat-containing protein 1 & $0.29 \pm 0.04$ & $0.02 \pm 0.01$ \\
\hline P28799 & GRN & Granulins & $0.3 \pm 0.03$ & $0.03 \pm 0.01$ \\
\hline Q96AE4 & FUBP1 & Far upstream element-binding protein 1 & $0.21 \pm 0.17$ & $0 \pm 0$ \\
\hline P38571 & LICH & Lysosomal acid lipase/cholesteryl ester hydrolase & $0.19 \pm 0.18$ & $0.02 \pm 0.02$ \\
\hline A8MXP9 & A8MXP9 & Matrin-3 & $0.23 \pm 0.14$ & $0 \pm 0$ \\
\hline 060885 & BRD4 & Bromodomain-containing protein 4 & $0.38 \pm 0.07$ & $0 \pm 0$ \\
\hline P52597 & HNRPF & Heterogeneous nuclear ribonucleoprotein $\mathrm{F}$ & $0.26 \pm 0.1$ & $0 \pm 0$ \\
\hline 043395 & PRPF3 & U4/U6 small nuclear ribonucleoprotein Prp3 & $0.32 \pm 0.03$ & $0 \pm 0$ \\
\hline Q9Y3B9 & RRP15 & RRP15-like protein & $0.28 \pm 0.09$ & $0.02 \pm 0.01$ \\
\hline 075643 & U520 & U5 small nuclear ribonucleoprotein $200 \mathrm{kDa}$ helicase & $0.38 \pm 0.05$ & $0 \pm 0$ \\
\hline Q13263 & TIF1B & Transcription intermediary factor 1 -beta & $0.22 \pm 0.17$ & $0 \pm 0$ \\
\hline Q8IX12 & CCAR1 & Cell division cycle and apoptosis regulator protein 1 & $0.4 \pm 0.08$ & $0 \pm 0$ \\
\hline Q9NZM5 & GSCR2 & Glioma tumor suppressor candidate region gene 2 protein & $0.22 \pm 0.18$ & $0.02 \pm 0.01$ \\
\hline 043290 & SNUT1 & U4/U6.U5 tri-snRNP-associated protein 1 & $0.28 \pm 0.11$ & $0.01 \pm 0.01$ \\
\hline Q13616 & CUL1 & Cullin-1 & $0.33 \pm 0.04$ & $0.01 \pm 0$ \\
\hline F8VUJ3 & F8VUJ3 & Polypeptide $\mathrm{N}$-acetylgalactosaminyltran sferase & $0.35 \pm 0.01$ & $0.01 \pm 0.02$ \\
\hline Q9NPL8 & TIDC1 & Complex I assembly factor TIMMDC1 & $0.3 \pm 0.07$ & $0.01 \pm 0.01$ \\
\hline Q10570 & CPSF1 & Cleavage and polyadenylation specificity factor subunit 1 & $0.32 \pm 0.06$ & $0 \pm 0$ \\
\hline Q96EP5 & DAZP1 & DAZ-associated protein 1 & $0.32 \pm 0.06$ & $0 \pm 0$ \\
\hline 075533 & SF3B1 & Splicing factor $3 \mathrm{~B}$ subunit 1 & $0.42 \pm 0.08$ & $0 \pm 0.01$ \\
\hline P26368 & U2AF2 & Splicing factor U2AF $65 \mathrm{kDa}$ subunit & $0.28 \pm 0.12$ & $0.01 \pm 0.01$ \\
\hline Q13523 & PRP4B & Serine/threonine-protein kinase PRP4 homolog & $0.35 \pm 0.03$ & $0.03 \pm 0.01$ \\
\hline F5H324 & F5H324 & Arylsulfatase E & $0.43 \pm 0.07$ & $0 \pm 0$ \\
\hline Q13283 & G3BP1 & Ras GTPase-activating protein-binding protein 1 & $0.38 \pm 0$ & $0.01 \pm 0.01$ \\
\hline Q16850 & CP51A & Lanosterol 14-alpha demethylase & $0.24 \pm 0.19$ & $0.03 \pm 0.01$ \\
\hline B7Z4B8 & B7Z4B8 & Heterogeneous nuclear ribonucleoprotein U-like protein 1 & $0.33 \pm 0.07$ & $0 \pm 0$ \\
\hline Q15459 & SF3A1 & Splicing factor $3 \mathrm{~A}$ subunit 1 & $0.41 \pm 0.03$ & $0 \pm 0$ \\
\hline Q96A72 & MGN2 & Protein mago nashi homolog 2 & $0.24 \pm 0.2$ & $0.01 \pm 0.01$ \\
\hline Q14152 & EIF3A & Eukaryotic translation initiation factor 3 subunit $\mathrm{A}$ & $0.38 \pm 0.01$ & $0 \pm 0$ \\
\hline P36578 & RL4 & $60 \mathrm{~S}$ ribosomal protein $\mathrm{L} 4$ & $0.34 \pm 0.07$ & $0 \pm 0$ \\
\hline 060506 & HNRPQ & Heterogeneous nuclear ribonucleoprotein Q & $0.28 \pm 0.16$ & $0 \pm 0$ \\
\hline Q8WVV9 & HNRLL & Heterogeneous nuclear ribonucleoprotein L-like & $0.31 \pm 0.11$ & $0.03 \pm 0.02$ \\
\hline Q14444 & CAPR1 & Caprin-1 & $0.33 \pm 0.1$ & $0.01 \pm 0.02$ \\
\hline Q92900 & RENT1 & Regulator of nonsense transcripts 1 & $0.3 \pm 0.14$ & $0.05 \pm 0$ \\
\hline 075448 & MED24 & Mediator of RNA polymerase II transcription subunit 24 & $0.36 \pm 0.06$ & $0.03 \pm 0.01$ \\
\hline Q00341 & VIGLN & Vigilin & $0.28 \pm 0.17$ & $0 \pm 0$ \\
\hline Q14789 & GOGB1 & Golgin subfamily B member 1 & $0.44 \pm 0.04$ & $0 \pm 0$ \\
\hline P55884-2 & EIF3B & Isoform 2 of Eukaryotic translation initiation factor 3 subunit B & $0.36 \pm 0.06$ & $0.04 \pm 0.01$ \\
\hline Q9UNX4 & WDR3 & WD repeat-containing protein 3 & $0.41 \pm 0$ & $0.01 \pm 0.02$ \\
\hline Q9NY61 & AATF & Protein AATF & $0.32 \pm 0.12$ & $0.01 \pm 0$ \\
\hline Q9H583 & HEAT1 & HEAT repeat-containing protein 1 & $0.38 \pm 0.05$ & $0.01 \pm 0$ \\
\hline P45973 & CBX5 & Chromobox protein homolog 5 & $0.44 \pm 0.03$ & $0.01 \pm 0$ \\
\hline Q9NV31 & IMP3 & U3 small nucleolar ribonucleoprotein protein IMP3 & $0.28 \pm 0.19$ & $0.03 \pm 0$ \\
\hline P46776 & RL27A & $60 \mathrm{~S}$ ribosomal protein $\mathrm{L} 27 \mathrm{a}$ & $0.32 \pm 0.14$ & $0.04 \pm 0$ \\
\hline Q92797 & SYMPK & Symplekin & $0.41 \pm 0.03$ & $0 \pm 0$ \\
\hline Q8NI36 & WDR36 & WD repeat-containing protein 36 & $0.46 \pm 0.04$ & $0 \pm 0$ \\
\hline Q9UKM9 & RALY & RNA-binding protein Raly & $0.38 \pm 0.07$ & $0 \pm 0$ \\
\hline P27105 & STOM & Erythrocyte band 7 integral membrane protein & $0.36 \pm 0.1$ & $0 \pm 0$ \\
\hline Q9UKD2 & MRT4 & mRNA turnover protein 4 homolog & $0.44 \pm 0.01$ & $0 \pm 0$ \\
\hline Q6YHK3 & CD109 & CD109 antigen & $0.36 \pm 0.11$ & $0.01 \pm 0$ \\
\hline Q12788 & TBL3 & Transducin beta-like protein 3 & $0.36 \pm 0.12$ & $0.01 \pm 0$ \\
\hline Q15029 & U5S1 & $116 \mathrm{kDa}$ U5 small nuclear ribonucleoprotein component & $0.42 \pm 0.04$ & $0 \pm 0$ \\
\hline Q9C0J8 & WDR33 & pre-mRNA 3' end processing protein WDR33 & $0.4 \pm 0.07$ & $0.03 \pm 0$ \\
\hline P11717 & MPRI & Cation-independent mannose-6-phosphate receptor & $0.43 \pm 0.03$ & $0.01 \pm 0$ \\
\hline Q9BYG3 & MK67I & MKI67 FHA domain-interacting nucleolar phosphoprotein & $0.31 \pm 0.2$ & $0.01 \pm 0$ \\
\hline Q12996 & CSTF3 & Cleavage stimulation factor subunit 3 & $0.35 \pm 0.15$ & $0.01 \pm 0$ \\
\hline B5MCF9 & B5MCF9 & Pescadillo homolog & $0.28 \pm 0.25$ & $0 \pm 0$ \\
\hline Q9H501 & ESF1 & ESF1 homolog & $0.31 \pm 0.21$ & $0.01 \pm 0$ \\
\hline Q92604 & LGAT1 & Acyl-CoA:lysophosphatidylglycerol acyltransferase 1 & $0.46 \pm 0.01$ & $0.03 \pm 0$ \\
\hline P15924 & DESP & Desmoplakin & $0.36 \pm 0.16$ & $0.01 \pm 0$ \\
\hline P53582 & MAP11 & Methionine aminopeptidase 1 & $0.35 \pm 0.18$ & $0.01 \pm 0$ \\
\hline 015230 & LAMA5 & Laminin subunit alpha- 5 & $0.4 \pm 0.11$ & $0.03 \pm 0$ \\
\hline Q9UNP9 & PPIE & Peptidyl-prolyl cis-trans isomerase $\mathrm{E}$ & $0.35 \pm 0.18$ & $0 \pm 0$ \\
\hline Q96ST2 & IWS1 & Protein IWS1 homolog & $0.4 \pm 0.11$ & $0.02 \pm 0$ \\
\hline Q9NVP1 & DDX18 & ATP-dependent RNA helicase DDX18 & $0.44 \pm 0.02$ & $0.05 \pm 0$ \\
\hline Q9BQ39 & DDX50 & ATP-dependent RNA helicase DDX50 & $0.40 \pm 0.08$ & $0.03 \pm 0$ \\
\hline P46777 & RL5 & $60 \mathrm{~S}$ ribosomal protein $\mathrm{L} 5$ & $0.36 \pm 0.10$ & $0.01 \pm 0$ \\
\hline Q8N684 & CPSF7 & Cleavage and polyadenylation specificity factor subunit 7 & $0.45 \pm 0.05$ & $0.03 \pm 0$ \\
\hline P18583-5 & SON & Isoform D of Protein SON & $0.40 \pm 0.09$ & $0.05 \pm 0$ \\
\hline 094776 & MTA2 & Metastasis-associated protein MTA2 & $0.38 \pm 0.12$ & $0 \pm 0$ \\
\hline P63010 & AP2B1 & AP-2 complex subunit beta & $0.34 \pm 0.15$ & $0.01 \pm 0$ \\
\hline
\end{tabular}


Protein-protein interaction network analysis

To further examine the comprehensive information obtained from the identified protein data, the PPI network was analyzed. The network model was generated using the Cytoscape web application based on information gained in up to 4 levels of functional analysis: fold-change of gene/protein expression, proteinprotein interactions, KEGG pathway enrichment and biological process enrichment. A merged network is shown in Fig. 7. Again, PPI analysis identified ribosome biogenesis in Table 4: The 50 apoptosis-associated proteins of enrichment analysis

\begin{tabular}{|c|c|c|c|c|}
\hline $\begin{array}{c}\text { Accession } \\
\text { NO. }\end{array}$ & Gene name & Protein name & $\begin{array}{l}\text { Fold change } \\
(\text { Mean } \pm \text { SD) }\end{array}$ & $\begin{array}{c}\text { P-value } \\
(\text { Mean } \pm S D)\end{array}$ \\
\hline P35232 & PHB & Prohibitin & $2.61 \pm 0.4$ & $0.01 \pm 0.02$ \\
\hline Q13464 & ROCK1 & Rho-associated protein kinase 1 & $2.75 \pm 0.61$ & $0 \pm 0$ \\
\hline P30101 & PDIA3 & Protein disulfide-isomerase $\mathrm{A} 3$ & $2.15 \pm 0.2$ & $0 \pm 0$ \\
\hline Q9BXJ9 & NAA15 & $\mathrm{N}$-alpha-acetyltransferase 15 , NatA auxiliary subunit & $2.1 \pm 0.1$ & $0 \pm 0$ \\
\hline P09874 & PARP1 & Poly [ADP-ribose] polymerase 1 & $2.48 \pm 0.46$ & $0 \pm 0.01$ \\
\hline P21796 & VDAC1 & Voltage-dependent anion-selective channel protein 1 & $3.01 \pm 0.17$ & $0 \pm 0$ \\
\hline Q07021 & C1QBP & Complement component $1 Q$ subcomponent-binding protein & $2.52 \pm 0.47$ & $0.03 \pm 0.01$ \\
\hline P45880 & VDAC2 & Voltage-dependent anion-selective channel protein 2 & $2.93 \pm 0.69$ & $0 \pm 0$ \\
\hline P07237 & PDIA1 & Protein disulfide-isomerase & $2.54 \pm 0.23$ & $0 \pm 0$ \\
\hline P05556 & ITB1 & Integrin beta-1 & $2.46 \pm 0.11$ & $0 \pm 0$ \\
\hline P48506 & GSH1 & Glutamate--cysteine ligase catalytic subunit & $2.49 \pm 0.03$ & $0.01 \pm 0.01$ \\
\hline P42224 & STAT1 & Signal transducer and activator of transcription 1-alpha/beta & $2.62 \pm 0.09$ & $0 \pm 0$ \\
\hline P49721 & PSB2 & Proteasome subunit beta type- 2 & $2.41 \pm 0.38$ & $0.03 \pm 0.02$ \\
\hline P11388 & TOP2A & DNA topoisomerase 2-alpha & $3.07 \pm 0.52$ & $0 \pm 0$ \\
\hline P12830 & CDH1 & Cadherin-1 & $2.30 \pm 0.10$ & $0.04 \pm 0.01$ \\
\hline P23229 & ITA6 & Integrin alpha-6 & $3.16 \pm 0.08$ & $0 \pm 0$ \\
\hline $\mathrm{P} 63104$ & $1433 Z$ & 14-3-3 protein zeta/delta & $2.71 \pm 0.77$ & $0 \pm 0$ \\
\hline 043707 & ACTN4 & Alpha-actinin- 4 & $6.61 \pm 1.84$ & $0 \pm 0$ \\
\hline P08758 & ANXA5 & Annexin A5 & $2.85 \pm 0.73$ & $0 \pm 0$ \\
\hline P08729 & $\mathrm{K} 2 \mathrm{C} 7$ & Keratin, type II cytoskeletal 7 & $4.32 \pm 0.78$ & $0 \pm 0$ \\
\hline P11387 & TOP1 & DNA topoisomerase 1 & $5.03 \pm 1.64$ & $0 \pm 0$ \\
\hline P30048 & PRDX3 & Thioredoxin-dependent peroxide reductase & $3.85 \pm 0.03$ & $0.02 \pm 0.01$ \\
\hline P14618 & KPYM & Pyruvate kinase PKM & $3.18 \pm 1.23$ & $0 \pm 0$ \\
\hline P23528 & COF1 & Cofilin-1 & $3.55 \pm 0.71$ & $0 \pm 0$ \\
\hline P00338 & LDHA & L-lactate dehydrogenase A chain & $3.09 \pm 1.42$ & $0 \pm 0$ \\
\hline Q8WUM4 & PDC6I & Programmed cell death 6 -interacting protein & $3.43 \pm 1.2$ & $0 \pm 0$ \\
\hline P21333 & FLNA & Filamin-A & $3.92 \pm 1.11$ & $0 \pm 0$ \\
\hline P15121 & ALDR & Aldose reductase & $3.84 \pm 1.92$ & $0 \pm 0$ \\
\hline Q92598 & HS105 & Heat shock protein $105 \mathrm{kDa}$ & $4.88 \pm 0.73$ & $0 \pm 0$ \\
\hline P05783 & K1C18 & Keratin, type I cytoskeletal 18 & $7.87 \pm 1.07$ & $0 \pm 0$ \\
\hline P04632 & CPNS1 & Calpain small subunit 1 & $6.43 \pm 0.96$ & $0.03 \pm 0.01$ \\
\hline P29317 & EPHA2 & Ephrin type-A receptor 2 & $6.39 \pm 2.2$ & $0 \pm 0$ \\
\hline P05787 & $\mathrm{K} 2 \mathrm{C} 8$ & Keratin, type II cytoskeletal 8 & $11.78 \pm 1.3$ & $0 \pm 0$ \\
\hline 060443 & DFNA5 & Non-syndromic hearing impairment protein 5 & $11.92 \pm 1.78$ & $0.03 \pm 0.01$ \\
\hline P02675 & FIBB & Fibrinogen beta chain & $0.32 \pm 0.07$ & $0 \pm 0$ \\
\hline P09601 & HMOX1 & Heme oxygenase 1 & $0.12 \pm 0.01$ & $0 \pm 0$ \\
\hline 000571 & DDX3X & ATP-dependent RNA helicase DDX3X & $0.32 \pm 0.07$ & $0.02 \pm 0.02$ \\
\hline Q9Y3A2 & UTP11 & Probable U3 small nucleolar RNA-associated protein 11 & $0.14 \pm 0.03$ & $0.04 \pm 0.01$ \\
\hline Q13573 & SNW1 & SNW domain-containing protein 1 & $0.12 \pm 0.05$ & $0.03 \pm 0.01$ \\
\hline 076021 & RL1D1 & Ribosomal L1 domain-containing protein 1 & $0.12 \pm 0.11$ & $0 \pm 0$ \\
\hline Q6P1J9 & CDC73 & Parafibromin & $0.27 \pm 0.03$ & $0 \pm 0$ \\
\hline Q14344 & GNA13 & Guanine nucleotide-binding protein subunit alpha- 13 & $0.39 \pm 0.12$ & $0.01 \pm 0.01$ \\
\hline Q86SJ2 & AMGO2 & Amphoterin-induced protein 2 & $0.25 \pm 0.08$ & $0 \pm 0$ \\
\hline Q8IX12 & CCAR1 & Cell division cycle and apoptosis regulator protein 1 & $0.4 \pm 0.08$ & $0 \pm 0$ \\
\hline Q9NZM5 & GSCR2 & Glioma tumor suppressor candidate region gene 2 protein & $0.22 \pm 0.18$ & $0.02 \pm 0.01$ \\
\hline 043290 & SNUT1 & U4/U6.U5 tri-snRNP-associated protein 1 & $0.28 \pm 0.11$ & $0.01 \pm 0.01$ \\
\hline Q13616 & CUL1 & Cullin-1 & $0.33 \pm 0.04$ & $0.01 \pm 0$ \\
\hline Q9NY61 & AATF & Protein AATF & $0.32 \pm 0.12$ & $0.01 \pm 0$ \\
\hline P11717 & MPRI & Cation-independent mannose-6-phosphate receptor & $0.43 \pm 0.03$ & $0.01 \pm 0$ \\
\hline P15924 & DESP & Desmoplakin & $0.36 \pm 0.16$ & $0.01 \pm 0$ \\
\hline
\end{tabular}

eukaryotes and the spliceosome as the most significantly enriched pathways. In the network, the proteins indicated with red circle nodes were up-regulated, and the proteins indicated with green circle nodes were downregulated. These data clearly show that most of the proteins were down-regulated in Ribosome biogenesis in eukaryotes, Spliceosome, and mRNA surveillance pathways, while the dysregulated proteins in Cysteine and methionine metabolism, Propanoate metabolism and Glycolysis/Gluconeogenesis were up-regulated.

\section{Evaluation of iTRAQ results using western blotting}

Based on the results of the MS analysis, the expression levels of five dysregulated proteins were validated using western blotting in TP-treated or negative control cells. The expression levels of two proteins (MTA2 and EIF4A3) were significantly down-regulated (Fig. 8A), while the expression levels of the remaining three proteins (PHB, CDH1 and AIFM1) were markedly increased in TP-treated cells compared with the corresponding control (Fig. 8B), consistent with the results from the MS analysis. Therefore, the altered expression levels of 312 proteins were considered induced by TP.

\section{Discussion}

TP has been widely investigated for its broad-spectrum anticancer activity. Many studies have shown that TP inhibits cell growth and induces apoptosis in various cancers, 
Fig. 5. GO enrichment analysis. An overview of the GO annotations of the 312 dysregulated proteins with up to 10 significantly enriched terms in three categories: biological process (BP), cellular component (CC) and molecular function (MF). The cutoff of P-value was set to 0.05 . Terms in the same category were ordered based on the P-values. Information for the percentages and numbers of involved genes/proteins in a term is provided on the left and right $y$-axes.

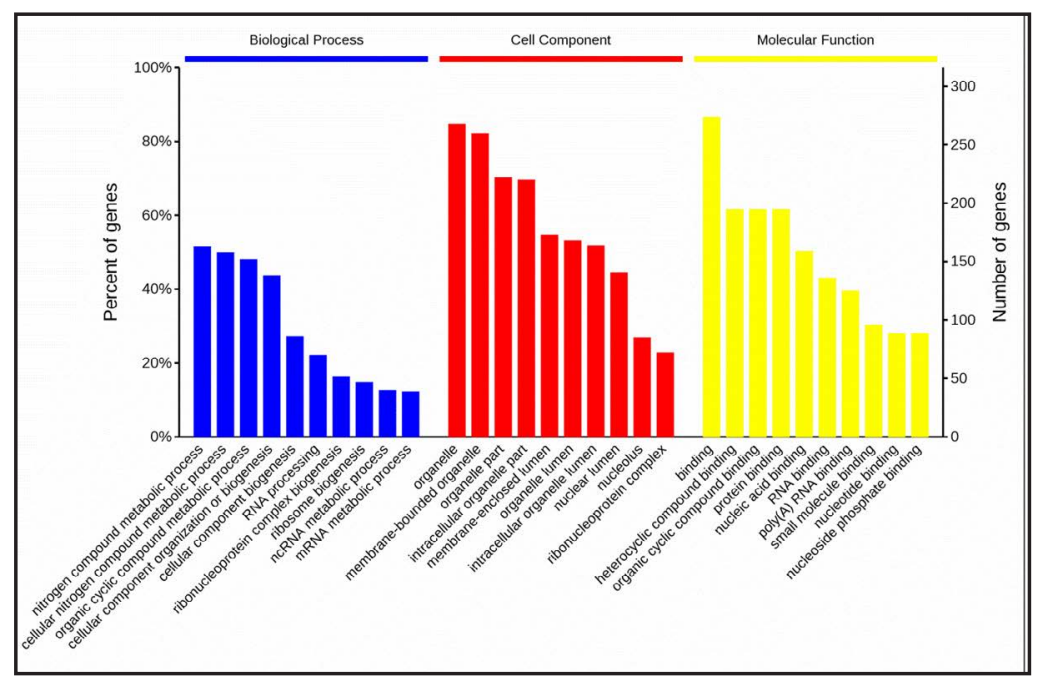

Fig. 6. Distribution of enriched KEGG pathways. Columns refer to related pathways, colored with gradient colors from midnight blue (smaller P-value) to lighter blue (larger P-value).

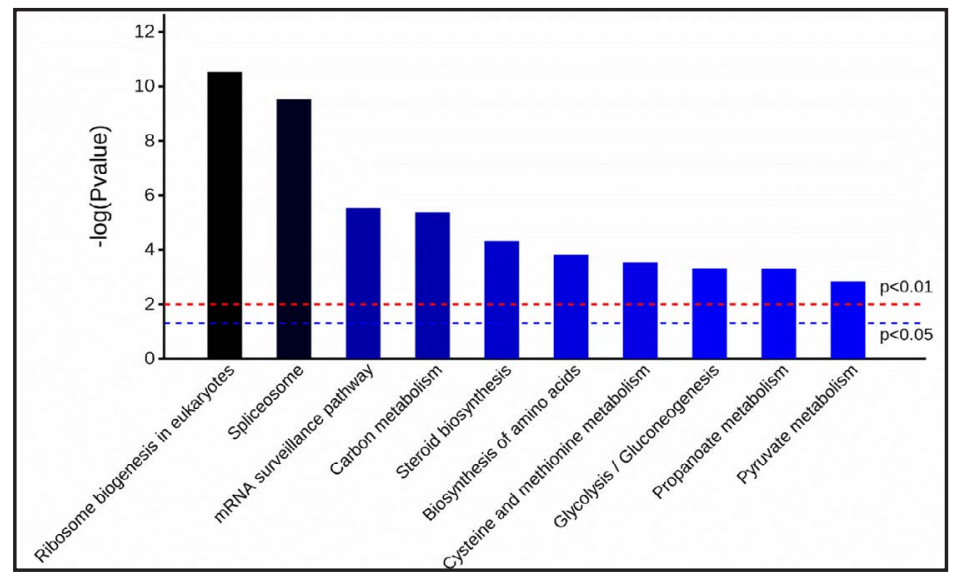

primarily through multiple mechanisms, including the suppression of various signaling pathways and proliferative and antiapoptotic factors in a given cell type and under specific conditions. TP has been reported to strongly inhibit the transcription of numerous proinflammatory mediators [25] and was also implicated as a potent inhibitor of NF-kappa B and a promoter of transcriptional arrest [26-30]. Recent studies have shown that TP inhibits RNA polymerase-mediated transcription by targeting transcription factors, leading to the down-regulation of certain mRNA molecules [31-33]. However, to our knowledge, there are no studies reporting the anti-proliferative and pro-apoptotic effects of TP against NSCLC cells at the proteomics level. In the present study, we attempted to investigate the potential protein targets of TP in a human NSCLC A549 lung adenocarcinoma cell line in vitro. First, we investigated the cytotoxicity of TP on A549 cells. TP strongly inhibited cell proliferation and induced cell apoptosis and cell cycle arrest in dose-dependent manners. Second, an iTRAQbased proteomics method was employed to analyze the molecular targets of A549 cancer cells after TP treatment, and pathway and network analyses were performed. Proteomics analysis is a powerful tool for the identification of biological markers and estimation of biological networks [34]. A global view of the inter-connectivity of signaling proteins and their actions is critically important for successful lung cancer therapy [35] and should provide a comprehensive perspective for elucidating the roles of TP as a potential agent for treatment of NSCLC. We observed 312 differentially expressed proteins in A549 cells after TP treatment. Moreover, bioinformatics analysis revealed that these proteins were involved in many BPs, including ribosome biogenesis, RNA processing, ribonucleoprotein 


\section{Cellular Physiology and Biochemistry Published \begin{tabular}{l|l} 
DO 2018 The Author(s). Published by S. Karger AG, Basel \\
www.karger.com/cpb
\end{tabular}

Fig. 7. Protein-protein interaction (PPI) network. The PPI analysis was based on fold changes of protein expression, PPIs, and KEGG pathway and biological process enrichments. Circle nodes refer to genes/proteins. The rectangles refer to KEGG pathways or biological processes, colored with gradient colors from yellow (smaller P-value) to blue (larger P-value). Genes/proteins are colored in red (up-regulation) and green (down-regulation). A default confidence cutoff of 400 was used: interactions with higher confidence scores are shown as solid lines between genes/proteins or are otherwise indicated as dashed lines.

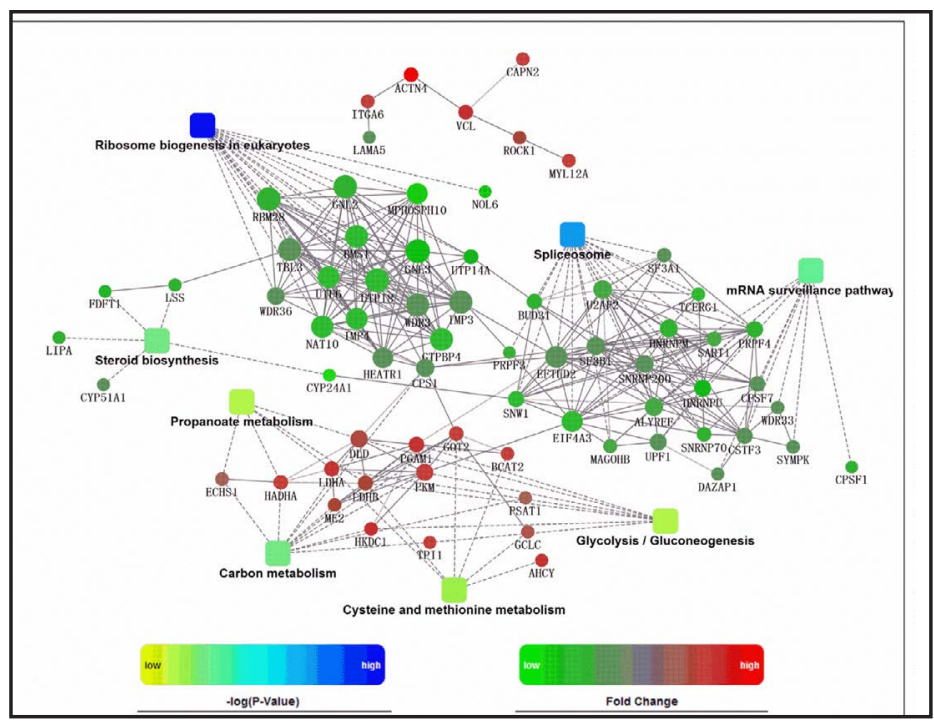

Fig. 8. Expression levels of the representative dysregulated proteins were verified using western blot analysis. (A) CDH1, AIFM1 and PHB are down-regulated proteins. (B) EIF4A3 and MTA2 are up-regulated proteins. $\beta$-actin was used as the loading control. Data are expressed as the means \pm SD $(\mathrm{n}=3) .{ }^{*} \mathrm{P}<0.05$ and ${ }^{* *} \mathrm{P}<0.01$ compared with the control group.

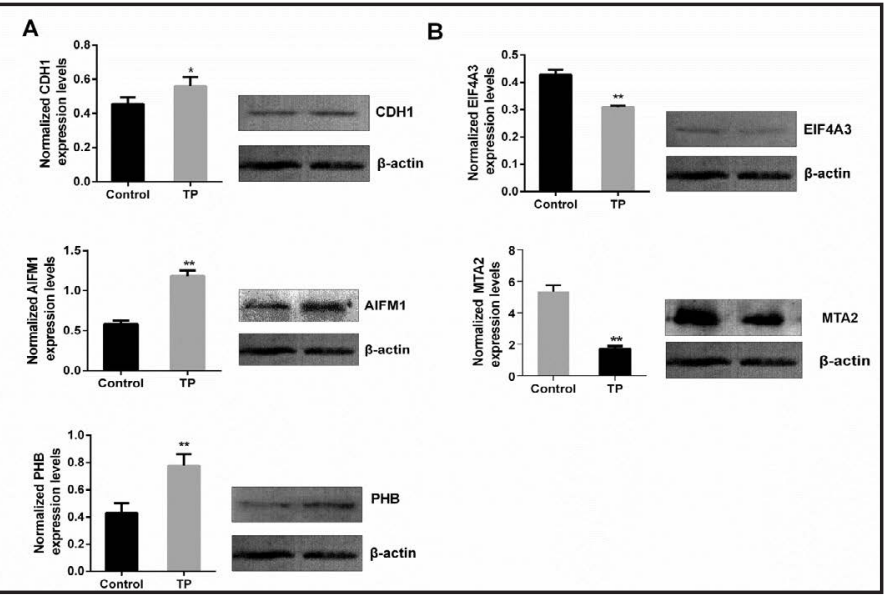

complex biogenesis, rRNA metabolic process, rRNA processing, ncRNA processing, cellular component biogenesis, and others. These proteins were implicated in 226 different KEGG pathways and associated with each other to form a network. The anticancer activity of TP against A549 cancer cells is mediated through its effects on multiple BPs and pathways, including ribosome biogenesis in eukaryotes, the spliceosome, mRNA surveillance pathway, PARP1/AIF pathway, metabolic pathway and other important molecular targets.

Ribosome biogenesis in eukaryotes and spliceosome and mRNA surveillance pathways are central processes for gene expression and protein synthesis, which are inextricably associated with cell growth and division. After TP treatment, most of the differentially expressed proteins involved in RNA metabolism pathways were significantly down-regulated. Among these down-regulated proteins, SNW1, HNRPM, EFTUD2, and SNRNP200 are components of the spliceosome. Many drugs have recently been demonstrated as inhibitors of RNA splicing, with cytotoxic effects on tumor cell lines [36]. SNW1 depletion induced apoptosis in breast cancer cells, and EFTUD2 knockdown also significantly promoted cellular apoptosis [37]. Previous studies have demonstrated that through the inhibition of the Akt signaling pathway, TP induces Bax- and Bcl-2-mediated mitochondrial apoptotic pathways, resulting in caspase-9- and caspase-3-triggered cell apoptosis [17, 38, 39]. In the present study, we identified key nuclear proteins and other mitochondrial proteins as down- or upstream targets of the Akt signaling pathway, such as REF1/Aly, GTPBP4, EIF4A3 and PHB. 
REF1/Aly, GTPBP4 and EIF4A3 are involved in RNA metabolism-related pathways. EIF4A3 (DDX48) is a core component of the exon junction complex (EJC) and plays a critical role in multiple posttranscriptional events, including RNA subcellular localization, nonsensemediated decay (NMD), and translation [40]. The AKT signaling pathway regulates the assembly of the core EJC proteins eIF4A3, MAGOH, and Y14 into complexes at speckled domains, which is essential for mRNA export. AKT inhibition results in the disorder of mRNA export and gene expression [41]. REF1/Aly is a nuclear speckle protein implicated in mRNA export and is a physiological target of the nuclear Akt signaling cascade [42, 43]. The depletion of Aly markedly blocks cell cycle progression and reduces cell growth and mRNA export, and these processes are regulated by Akt phosphorylation [43, 44]. GTPBP4, located in the nucleus, is involved in the biosynthesis of the 60S subunit of the ribosome [45] and can be used as a molecular switch to control signal transduction pathways, protein synthesis and other biological processes. GTPBP4 down-regulation in colorectal cancer cell lines significantly inhibited cell proliferation [46]. GTPBP4 is closely associated with the MAPK signaling pathway and participates in the regulation of MAPK and Akt signaling pathways through interactions with AKT $[47,48]$. It has also been reported that GTPBP4 binds to P53, and low GTPBP4 expression leads to the aggregation and activation of P53 proteins, which regulate the downstream apoptosis-related factors caspase-3, caspase-9, and PARP [49]. Prohibitin belongs to the Band-7 protein family and is widely present in different cellular compartments. Several studies using different organism models have provided strong evidence for critical biological roles of PHB in mitochondrial function, cell proliferation, and development. The contribution of PHB to cancer cell apoptosis may depend on the stimuli and cell type [50]. Recent studies have characterized PHB as a multifunctional protein involved in the PI3K/Akt and Ras/MAPK/ERK signaling pathways. A recent study in cancer cells showed that Akt phosphorylates PHB at Thr258. PHB could also indirectly facilitate crosstalk between the PI3K/Akt and Ras/MAPK/ERK pathways through interactions with their signaling intermediates. The emerging roles of PHB in the PI3K/Akt and Ras/ERK pathways highlight the importance of PHB in the crosstalk between signaling pathways. In the present study, PHB was up-regulated in the TP-induced apoptosis of NSCLC cells. We speculate that PHB may play an important role in the TP-induced apoptosis of A549 cells. Targeting PHB may have inspiring prospects in the future research of TP anti-tumor activity.

As described above, we speculated that TP-induced toxicities in lung cancer cells may be associated with the inhibition of RNA metabolism-related pathways, regulated by the Akt signaling pathway via important molecular target proteins. TP inhibits global ribosome biogenesis and splicing in cancer cells, which may explain the high potency of TP in killing lung cancer. Further studies are required to determine the potential link between the target proteins induced by TP and lung cancer and to provide a new strategy for cancer therapy.

Apoptosis-inducing factor 1 (AIFM1) is a mitochondrial flavoprotein with a critical role in programmed cell death. AIFM1 is a cell death executioner alternative to caspases [51]. Distinguishing from classic caspase-dependent apoptosis, the PARP1/AIF death cascade is a highly orchestrated and caspase-independent programmed cell death process termed parthanatos [52, 53]. In the present study, both AIF and PARP1 were up-regulated in NSCLC A549 cells after TP treatment, indicating that in addition to the classic caspase-dependent apoptosis pathway [17], the PARP1/AIF pathway may be another mechanism for TP to induce lung cancer cell apoptosis.

Unlike normal human cells, cancer cells display metabolic reprogramming to meet cell growth and proliferation needs [54]. Altered metabolic pathways in cancer cells may be attractive targets for anticancer therapy [55]. In the present study, some proteins related to metabolic pathways were dysregulated after TP treatment. The dysregulated proteins ECHS1, HADHA, PKM, AHCY and GOT2 are located in the mitochondria, and these enzymes are involved in apoptosis initiation and development. The mitochondrion is an important organelle with multiple functions, including ATP production, lipid metabolism, developmental processes and apoptosis regulation [56]. The expression levels of these proteins were enhanced during TP-induced apoptosis, suggesting that apoptosis is an active

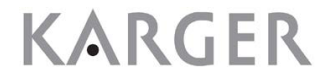




\section{Cellular Physiology Cell Physiol Biochem 2018;45:917-934

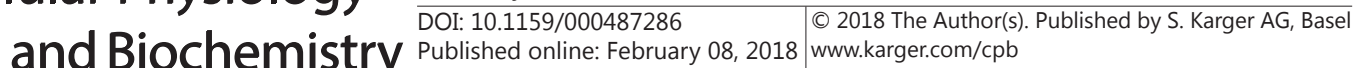

and energy-consuming procedure. These results demonstrated that energy metabolism is important in TP-induced apoptosis. Further research is required to determine the potential link between the altered mitochondrial enzymes induced by TP and NSCLC and to provide a new strategy for NSCLC therapy.

Metastasis is the ultimate cause of death for most cancer patients. TP has been reported as an inhibitor of lung cancer cell migration and metastasis, but its mechanism is not clearly defined $[12,57,58]$. We observed some other differentially expressed proteins, such as MTA2 and E-cadherin (CDH1), implicated in cancer cell migration. MTA2 is a member of the metastasis tumor-associated family of transcriptional regulators and acts as a central regulator of key gene expression pathways central to metastatic dissemination [59]. MTA2 knockdown in human cancer cells significantly inhibited migration and invasion [60]. E-cadherin is the core protein of the epithelial adherens junction. The loss of E-cadherin expression is a crucial step in the epithelial-mesenchymal transition (EMT) and is involved in cancer invasion and metastasis [61]. In human tumors, E-cadherin down-regulation is frequently associated with poor prognosis $[62,63]$. Interestingly, MTA2 promotes NSCLC metastasis through E-cadherin inhibition [64]. In the present study, we observed that TP not only up-regulates E-cadherin but also down-regulates MTA2, which might be a new target of the TP-mediated inhibition of A549 cell migration.

In conclusion, TP showed significant cytotoxicity in human A549 lung cancer cells, induced cell apoptosis and blocked cell cycle arrest. Potential cytotoxicity mechanisms were explored using an iTRAQ-based proteomics approach. The results provided the first evidence that the broad-spectrum anti-tumor activity of TP in lung cancer cells may be associated with inhibition of RNA metabolism and protein synthesis. Among the large number of differentially expressed proteins identified, some proteins, which may be potential targets for lung cancer treatment in the future, were validated. The present study provides an effective platform for the anticancer activity of TP. However, the present study has several limitations. First, there is a lack of the same experiments on normal lung cells. Second, protein profile changes in normal lung cells in response to TP may better reveal the specificity of TP effects on cancer cells. Moreover, the in vivo activity, clinical application, and other mechanisms of TP against NSCLC require further investigation.

\section{Abbreviations}

TP (triptolide); PNCA-1 (pancreatic cancer cells); NSCLC (non-small cell lung cancer); iTRAQ (isobaric tags for relative and absolute quantitation); NanoLC-MS/MS (nano liquid chromatography-mass spectrometry); FBS (fetal bovine serum); DMSO (dimethylsulfoxide); BCA (bicinchoninic acid); PI (propidium iodide); PMSF (phenylmethanesulfonyl fluoride); BP (biological process); CC (cellular component); KEGG (Kyoto Encyclopedia of Genes and Genomes); MF (molecular function); SDS-PAGE (sodium dodecyl sulfate-polyacrylamide gel electrophoresis); PVDF (polyvinylidene fluoride); GO (Gene Ontology); HP-RP (high-pH reverse phase); CE (collision energy); FDR (false discovery rate.);

\section{Acknowledgements}

This study was financially supported by grants from the Science Foundation from the Science and Technology Project of Zhejiang Province (2014F10014) and the Natural Science Foundation of Zhejiang Province (Q17H290005). This study was also supported by National Science Foundation of China (No. 81774026).

\section{Disclosure Statement}

No conflicts of interest exist in the submission of this manuscript, and this manuscript was approved by all authors for publication. 


\section{Cellular Physiology Cell Physiol Biochem 2018;45:917-934 \begin{tabular}{l|l|l} 
and Biochemistry Published online: February 08, 2018 & $\begin{array}{l}\text { (c) } 2018 \text { The Author(s). Published by S. Karger AG, Basel } \\
\text { www.karger.com/cpb }\end{array}$ \\
\hline
\end{tabular}}

Li et al.: Proteomics Analysis of Triptolide Targets in A549 Cells

\section{References}

1 Torre LA, Siegel RL, Jemal A: Lung Cancer Statistics. Adv Exp Med Biol 2016;893:1-19.

2 Mizuno K, Mataki H, Seki N, Kumamoto T, Kamikawaji K, Inoue H: MicroRNAs in non-small cell lung cancer and idiopathic pulmonary fibrosis. J Hum Genet 2016.

-3 Deng W, Yan M, Yu T, Ge H, Lin H, Li J, Liu Y, Geng Q, Zhu M, Liu L, He X, Yao M: Quantitative proteomic analysis of the metastasis-inhibitory mechanism of miR-193a-3p in non-small cell lung cancer. Cell Physiol Biochem 2015;35:1677-1688.

4 Rajeswaran A, Trojan A, Burnand B, Giannelli M: Efficacy and side effects of cisplatin- and carboplatinbased doublet chemotherapeutic regimens versus non-platinum-based doublet chemotherapeutic regimens as first line treatment of metastatic non-small cell lung carcinoma: a systematic review of randomized controlled trials. Lung Cancer 2008;59:1-11.

5 Kupchan SM, Court WA, Dailey RG, Jr., Gilmore CJ, Bryan RF: Triptolide and tripdiolide, novel antileukemic diterpenoid triepoxides from Tripterygium wilfordii. J Am Chem Soc 1972;94:7194-7195.

-6 Corson TW, Crews CM: Molecular understanding and modern application of traditional medicines: triumphs and trials. Cell 2007;130:769-774.

7 Sun S, Wang Y, Zhou Y: [Research progress on immunosuppressive activity of monomers extracted from Chinese medicine]. Zhongguo Zhong Yao Za Zhi 2010;35:393-396.

8 Ziaei S, Halaby R: Immunosuppressive, anti-inflammatory and anti-cancer properties of triptolide: A mini review. Avicenna J Phytomed 2016;6:149-164.

-9 Li J, Liu R, Yang Y, Huang Y, Li X, Liu R, Shen X: Triptolide-induced in vitro and in vivo cytotoxicity in human breast cancer stem cells and primary breast cancer cells. Oncol Rep 2014;31:2181-2186.

10 Mujumdar N, Banerjee S, Chen Z, Sangwan V, Chugh R, Dudeja V, Yamamoto M, Vickers SM, Saluja AK: Triptolide activates unfolded protein response leading to chronic ER stress in pancreatic cancer cells. Am J Physiol Gastrointest Liver Physiol 2014;306:G1011-1020.

11 Wang Y, Liu T, Li H: Enhancement of triptolide-loaded micelles on tumorigenicity inhibition of human ovarian cancer. J Biomater Sci Polym Ed 2016;27:545-556.

12 Reno TA, Kim JY, Raz DJ: Triptolide Inhibits Lung Cancer Cell Migration, Invasion, and Metastasis. Ann Thorac Surg 2015;100:1817-1824; discussion 1824-1815.

13 Zhao F, Huang W, Zhang Z, Mao L, Han Y, Yan J, Lei M: Triptolide induces protective autophagy through activation of the CaMKKbeta-AMPK signaling pathway in prostate cancer cells. Oncotarget 2016;7:53665382.

14 Nakazato T, Sagawa M, Kizaki M: Triptolide induces apoptotic cell death of multiple myeloma cells via transcriptional repression of Mcl-1. Int J Oncol 2014;44:1131-1138.

15 Cheng X, Shi W, Zhao C, Zhang D, Liang P, Wang G, Lu L: Triptolide sensitizes human breast cancer cells to tumor necrosis factoralphainduced apoptosis by inhibiting activation of the nuclear factorkappaB pathway. Mol Med Rep 2016;13:3257-3264.

16 Li Y, Hu S: Triptolide sensitizes liver cancer cell lines to chemotherapy in vitro and in vivo. Panminerva Med 2014;56:211-220.

17 Meng G, Wang W, Chai K, Yang S, Li F, Jiang K: Combination treatment with triptolide and hydroxycamptothecin synergistically enhances apoptosis in A549 lung adenocarcinoma cells through PP2A-regulated ERK, p38 MAPKs and Akt signaling pathways. Int J Oncol 2015;46:1007-1017.

18 Seo J, Lee KJ: Post-translational modifications and their biological functions: proteomic analysis and systematic approaches. J Biochem Mol Biol 2004;37:35-44.

19 Blackstock WP, Weir MP: Proteomics: quantitative and physical mapping of cellular proteins. Trends Biotechnol 1999;17:121-127.

20 Usami M, Mitsunaga K: Proteomic analysis and in vitro developmental toxicity tests for mechanism-based safety evaluation of chemicals. Expert Rev Proteomics 2011;8:153-155.

21 Huang Q Zhang J, Peng S, Du M, Ow S, Pu H, Pan C, Shen H: Proteomic analysis of perfluorooctane sulfonateinduced apoptosis in human hepatic cells using the iTRAQ technique. J Appl Toxicol 2014;34:1342-1351.

22 Chen H, Xu L, Yin L, Xu Y, Han X, Qi Y, Zhao Y, Liu K, Peng J: iTRAQ-based proteomic analysis of dioscin on human HCT-116 colon cancer cells. Proteomics 2014;14:51-73.

23 Zieske LR: A perspective on the use of ITRAQ reagent technology for protein complex and profiling studies. J Exp Bot 2006;57:1501-1508. 


\section{Cellular Physiology Cell Physiol Biochem 2018;45:917-934 \begin{tabular}{l|l|l} 
and Biochemistry 2018 The Author(s). Published by S. Karger AG, Basel & (c) 10.1159/000487286 \\
wuww.karger.com/cpb
\end{tabular}

24 Ma S, Sun Y, Zhao X, Xu P: [Recent advance in high accuracy iTRAQ for quantitative proteomics]. Sheng Wu Gong Cheng Xue Bao 2014;30:1073-1082.

25 Wu Y, Cui J, Bao X, Chan S, Young DO, Liu D, Shen P: Triptolide attenuates oxidative stress, NF-kappaB activation and multiple cytokine gene expression in murine peritoneal macrophage. Int J Mol Med 2006;17:141-150.

-26 Leuenroth SJ, Crews CM: Triptolide-induced transcriptional arrest is associated with changes in nuclear substructure. Cancer Research 2008;68:5257-5266.

27 Oliveira AR, Beyer G, Chugh R, Skube SJ, Majumder K, Banerjee S, Sangwan V, Li L, Dawra RK, Subramanian $\mathrm{S}$, Saluja AK, Dudeja V: Triptolide abrogates growth of colon cancer and induces cell cycle arrest by inhibiting transcriptional activation of E2F. Lab Invest 2015;95:648-659.

28 Park SW, Kim YI: Triptolide induces apoptosis of PMA-treated THP-1 cells through activation of caspases, inhibition of NF-kappaB and activation of MAPKs. Int J Oncol 2013;43:1169-1175.

29 Ou CC, Chen YW, Hsu SC, Sytwu HK, Loh SH, Li JW, Liu JY: Triptolide Transcriptionally Represses HER2 in Ovarian Cancer Cells by Targeting NF-kappaB. Evid Based Complement Alternat Med 2012;2012:350239.

30 Chen M, Shi JT, Lv ZQ, Huang LJ, Lin XL, Zhang W, Liang RY, Li YQ Jiang SP: Triptolide inhibits TGF-beta1 induced proliferation and migration of rat airway smooth muscle cells by suppressing NF-kappaB but not ERK1/2. Immunology 2014.

-31 Huang M, Zhang H, Liu T, Tian D, Gu L, Zhou M: Triptolide inhibits MDM2 and induces apoptosis in acute lymphoblastic leukemia cells through a p53-independent pathway. Mol Cancer Ther 2013;12:184-194.

32 Titov DV, Gilman B, He QL, Bhat S, Low WK, Dang Y, Smeaton M, Demain AL, Miller PS, Kugel JF, Goodrich JA, Liu JO: XPB, a subunit of TFIIH, is a target of the natural product triptolide. Nat Chem Biol 2011;7:182-188.

-33 Vispe S, DeVries L, Creancier L, Besse J, Breand S, Hobson DJ, Svejstrup JQ Annereau JP, Cussac D, Dumontet C, Guilbaud N, Barret JM, Bailly C: Triptolide is an inhibitor of RNA polymerase I and IIdependent transcription leading predominantly to down-regulation of short-lived mRNA. Mol Cancer Ther 2009;8:2780-2790.

34 da Costa JP, Carvalhais V, Ferreira R, Amado F, Vilanova M, Cerca N, Vitorino R: Proteome signatures-how are they obtained and what do they teach us? Applied Microbiology and Biotechnology 2015;99:74177431.

35 Pe'er D, Hacohen N: Principles and strategies for developing network models in cancer. Cell 2011;144:864873.

36 Bonnal S, Vigevani L, Valcarcel J: The spliceosome as a target of novel antitumour drugs. Nat Rev Drug Discov 2012;11:847-859.

-37 Sato N, Maeda M, Sugiyama M, Ito S, Hyodo T, Masuda A, Tsunoda N, Kokuryo T, Hamaguchi M, Nagino M, Senga T: Inhibition of SNW1 association with spliceosomal proteins promotes apoptosis in breast cancer cells. Cancer Med 2015;4:268-277.

-38 Kim MJ, Lee TH, Kim SH, Choi YJ, Heo J, Kim YH: Triptolide inactivates Akt and induces caspase-dependent death in cervical cancer cells via the mitochondrial pathway. Int J Oncol 2010;37:1177-1185.

-39 Xie CQ Zhou P, Zuo J, Li X, Chen Y, Chen JW: Triptolide exerts pro-apoptotic and cell cycle arrest activity on drug-resistant human lung cancer A549/Taxol cells via modulation of MAPK and PI3K/Akt signaling pathways. Oncol Lett 2016;12:3586-3590.

40 Chan CC, Dostie J, Diem MD, Feng W, Mann M, Rappsilber J, Dreyfuss G: eIF4A3 is a novel component of the exon junction complex. RNA 2004;10:200-209.

41 Quaresma AJ, Sievert R, Nickerson JA: Regulation of mRNA export by the PI3 kinase/AKT signal transduction pathway. Mol Biol Cell 2013;24:1208-1221.

42 Zhou Z, Luo MJ, Straesser K, Katahira J, Hurt E, Reed R: The protein Aly links pre-messenger-RNA splicing to nuclear export in metazoans. Nature 2000;407:401-405.

43 Okada M, Jang SW, Ye K: Akt phosphorylation and nuclear phosphoinositide association mediate mRNA export and cell proliferation activities by ALY. Proc Natl Acad Sci U S A 2008;105:8649-8654.

44 Stubbs SH, Conrad NK: Depletion of REF/Aly alters gene expression and reduces RNA polymerase II occupancy. Nucleic Acids Res 2015;43:504-519.

-45 Fuentes JL, Datta K, Sullivan SM, Walker A, Maddock JR: In vivo functional characterization of the Saccharomyces cerevisiae 60S biogenesis GTPase Nog1. Mol Genet Genomics 2007;278:105-123.

-46 Yu H, Jin S, Zhang N, Xu Q: Up-regulation of GTPBP4 in colorectal carcinoma is responsible for tumor metastasis. Biochem Biophys Res Commun 2016;480:48-54. 


\section{Cellular Physiology Cell Physiol Biochem 2018;45:917-934

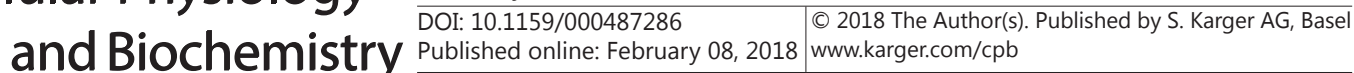

Li et al.: Proteomics Analysis of Triptolide Targets in A549 Cells

47 Hu ZW, Shi XY, Lin RZ, Hoffman BB: Contrasting signaling pathways of alpha1A- and alpha1B-adrenergic receptor subtype activation of phosphatidylinositol 3-kinase and Ras in transfected NIH3T3 cells. Mol Endocrinol 1999;13:3-14.

48 Uberall F, Hellbert K, Kampfer S, Maly K, Villunger A, Spitaler M, Mwanjewe J, Baier-Bitterlich G, Baier G, Grunicke HH: Evidence that atypical protein kinase C-lambda and atypical protein kinase C-zeta participate in Ras-mediated reorganization of the F-actin cytoskeleton. J Cell Biol 1999;144:413-425.

49 Lunardi A, Di Minin G, Provero P, Dal Ferro M, Carotti M, Del Sal G, Collavin L: A genome-scale protein interaction profile of Drosophila p53 uncovers additional nodes of the human p53 network. Proc Natl Acad Sci U S A 2010;107:6322-6327.

50 Peng YT, Chen P, Ouyang RY, Song L: Multifaceted role of prohibitin in cell survival and apoptosis. Apoptosis 2015;20:1135-1149.

51 Cimadamore F, Curchoe CL, Alderson N, Scott F, Salvesen G, Terskikh AV: Nicotinamide rescues human embryonic stem cell-derived neuroectoderm from parthanatic cell death. Stem Cells 2009;27:1772-1781.

52 Hong SJ, Dawson TM, Dawson VL: Nuclear and mitochondrial conversations in cell death: PARP-1 and AIF signaling. Trends Pharmacol Sci 2004;25:259-264.

53 Yu SW, Andrabi SA, Wang H, Kim NS, Poirier GG, Dawson TM, Dawson VL: Apoptosis-inducing factor mediates poly(ADP-ribose) (PAR) polymer-induced cell death. Proc Natl Acad Sci U S A 2006;103:1831418319.

54 Dang NH, Singla AK, Mackay EM, Jirik FR, Weljie AM: Targeted cancer therapeutics: biosynthetic and energetic pathways characterized by metabolomics and the interplay with key cancer regulatory factors. Curr Pharm Des 2014;20:2637-2647.

55 Masoudi-Nejad A, Asgari Y: Metabolic cancer biology: structural-based analysis of cancer as a metabolic disease, new sights and opportunities for disease treatment. Semin Cancer Biol 2015;30:21-29.

56 Fulda S, Galluzzi L, Kroemer G: Targeting mitochondria for cancer therapy. Nat Rev Drug Discov 2010;9:447-464.

57 Yang S, Chen J, Guo Z, Xu XM, Wang L, Pei XF, Yang J, Underhill CB, Zhang L: Triptolide inhibits the growth and metastasis of solid tumors. Mol Cancer Ther 2003;2:65-72.

58 Sangwan V, Banerjee S, Jensen KM, Chen Z, Chugh R, Dudeja V, Vickers SM, Saluja AK: Primary and liver metastasis-derived cell lines from KrasG12D; Trp53R172H; Pdx-1 Cre animals undergo apoptosis in response to triptolide. Pancreas 2015;44:583-589.

59 Covington KR, Fuqua SA: Role of MTA2 in human cancer. Cancer Metastasis Rev 2014;33:921-928.

60 Zhou C, Ji J, Cai Q, Shi M, Chen X, Yu Y, Liu B, Zhu Z, Zhang J: MTA2 promotes gastric cancer cells invasion and is transcriptionally regulated by Sp1. Mol Cancer 2013;12:102.

-61 Vergara D, Simeone P, Latorre D, Cascione F, Leporatti S, Trerotola M, Giudetti AM, Capobianco L, Lunetti P, Rizzello A, Rinaldi R, Alberti S, Maffia M: Proteomics analysis of E-cadherin knockdown in epithelial breast cancer cells. J Biotechnol 2015;202:3-11.

62 Pannone G, Santoro A, Feola A, Bufo P, Papagerakis P, Lo Muzio L, Staibano S, Ionna F, Longo F, Franco R, Aquino G, Contaldo M, De Maria S, Serpico R, De Rosa A, Rubini C, Papagerakis S, Giovane A, Tombolini V, Giordano A, Caraglia M, Di Domenico M: The role of E-cadherin down-regulation in oral cancer: CDH1 gene expression and epigenetic blockage. Curr Cancer Drug Targets 2014;14:115-127.

63 Mareel MM, Behrens J, Birchmeier W, De Bruyne GK, Vleminckx K, Hoogewijs A, Fiers WC, Van Roy FM: Down-regulation of E-cadherin expression in Madin Darby canine kidney (MDCK) cells inside tumors of nude mice. Int J Cancer 1991;47:922-928.

64 Zhang B, Zhang H, Shen G: Metastasis-associated protein 2 (MTA2) promotes the metastasis of non-smallcell lung cancer through the inhibition of the cell adhesion molecule Ep-CAM and E-cadherin. Jpn J Clin Oncol 2015;45:755-766. 